\title{
Systèmes rythmiques, métissages et enjeux symboliques des musiques d'Amérique latine
}

\section{Michel Plisson}

\section{(2) OpenEdition}

1 Journals

Édition électronique

URL : http://journals.openedition.org/ethnomusicologie/1963

ISSN : 2235-7688

\section{Éditeur}

ADEM - Ateliers d'ethnomusicologie

Édition imprimée

Date de publication : 1 janvier 2001

Pagination : 23-54

ISBN : 2-8257-0723-6

ISSN : $1662-372 X$

\section{Référence électronique}

Michel Plisson, «Systèmes rythmiques, métissages et enjeux symboliques des musiques d'Amérique latine », Cahiers d'ethnomusicologie [En ligne], 13 | 2001, mis en ligne le 17 décembre 2012, consulté le 10 décembre 2020. URL : http://journals.openedition.org/ethnomusicologie/1963

Ce document a été généré automatiquement le 10 décembre 2020.

Article L.111-1 du Code de la propriété intellectuelle. 


\title{
Systèmes rythmiques, métissages et enjeux symboliques des musiques d'Amérique latine
}

\author{
Michel Plisson
}

1 En Amérique latine, les musiques traditionnelles se sont constituées durant cinq siècles à partir d'éléments culturels étrangers les uns aux autres, produisant des réactions alchimiques complexes. De nouvelles expressions musicales sont nées de ces brassages dont la résultante la plus achevée est le genre musico-chorégraphique. Au delà de cet entrelacs multiple et foisonnant, nous sommes tentés de chercher les liens organiques unissant ces genres musicaux entre eux. Notre réflexion n'est pas tant centrée sur les métissages que sur les processus d'adaptation sociale qui ont conduit à la production de musiques nouvelles. En étudiant les conditions sociales qui présidèrent à leur éclosion, nous tenterons d'établir des relations de parenté entre ces musiques et de découvrir une éventuelle logique organique interne derrière leur extrême diversité.

2 Nous ne chercherons donc pas à dresser un inventaire complet, ni à procéder à une analyse tant soit peu exhaustive des genres musico-chorégraphiques d'Amérique latine dont l'effectif total dépasse probablement le demi-millier, sans compter les formes régionales ou locales ${ }^{1}$. Nous tenterons seulement d'appréhender certains caractères originaux propres aux musiques traditionnelles de cette partie du monde et de dégager quelques lignes de force conduisant à des syncrétismes musicaux, notamment rythmiques.

3 Tout d'abord, nous envisagerons quelques considérations théoriques quant aux interactions entre les groupes sociaux, d'où procède le métissage. Ensuite, nous tenterons d'évaluer dans quelle mesure les genres musicaux représentent symboliquement des enjeux à la fois identitaires et contradictoires entre groupes sociaux. Une fois constitué, le genre musical comme produit de métissage, acquiert des caractères musicaux spécifiques qui occultent pour partie ses éléments d'origine. Enfin, nous analyserons deux grands patrons rythmiques originaux issus des syncrétismes rythmiques d'une part d'origine 
hispanique, et d'autre part d'origine africaine, à partir desquels se sont élaborés, en Amérique latine, des centaines de genres musico-chorégraphiques.

4 Nous écartons délibérément ici les musiques amérindiennes, notamment celles issues des cultures andines, qui constituent la troisième grande famille musicale. Nous laissons de côté également d'autres musiques, souvent très populaires, plus influencées par des courants provenant d'Amérique du Nord, dont la diffusion dans la partie sud du continent a été en progression croissante à partir des années quatre-vingt, diminuant considérablement le champ d'action social des musiques plus traditionnelles.

\section{Métissages musicaux et relations dominant-dominé}

Il est aujourd'hui banal d'affirmer que les musiques d'Amérique latine sont le produit syncrétique des trois familles culturelles : amérindienne, européenne et africaine. Il est nécessaire d'ajouter que ces trois familles culturelles se sont entremêlées de façon contradictoire. Partout, l'élément européen a été dominant. Il s'est imposé aux autres cultures par la force, avec la violence et la brutalité que l'histoire a révélées. «L'élément européen ", c'est la culture du maître blanc contre celle de l'Indien, du Noir, puis plus tard, du Métis, du Zambo, du Mulato. Le métissage s'est construit sur des cultures amérindiennes disloquées, puis sur des cultures africaines reconstruites.

6 L'identité culturelle de la société coloniale s'est édifiée sur la base de relations dominantdominé. C'est donc de façon contradictoire que les métissages se sont effectués. Les musiques se sont adaptées à ces conditions subies. Dans les Andes, les modes musicaux ont intégré des échelles musicales contenant des degrés supplémentaires, enrichissant ainsi le corpus mélodique. Les instruments européens ont trouvé un lieu privilégié d'acclimatation. Introduits dès le premiers temps de la conquête, ils ont proliféré avec bonheur et ont généré en Amérique latine des familles entières de cordophones. Des corpus nouveaux tant poétiques que mélodiques se sont créés.

7 Ces métissages ne furent pas l'œuvre du corps social dans son ensemble, mais presque toujours celle des couches populaires, elles-mêmes aux confluents de plusieurs cultures.

8 Les Européens qui, à l'époque coloniale, sillonnèrent les Amériques, nous renseignent sur le contexte social des pratiques musicales, au-delà des préjugés dont ils sont presque toujours empreints. Ces voyageurs - comme on les nomme - relayant les chroniqueurs du premier siècle de la conquête, relatent les fêtes auxquelles ils assistent dans les villages, cabarets ou parties communes des haciendas. Ces journaux de voyage désignent constamment les couches les plus populaires comme ayant la pratique musicale la plus intense. Les témoignages sont nombreux : Max Radiguet, cité par Carlos Vega ${ }^{2}$, observe au Pérou en 1841 que « Les Noirs, surtout, déforment les danses gracieuses et enflammées du Pérou, introduisant dans celles-ci les postures grotesques et les mouvements désordonnés de leurs bamboulas africaines $»^{3}$.

Ces mêmes voyageurs déplorent presque toujours le saccage des belles danses européennes. Un certain Emile Carrey voyageant à Lima en 1875 décrit ainsi une danse parmi les Noirs: "Mais la musique devient chaque fois plus vive, et leurs propres mouvements les enivrent. Leurs membres s'agitent jusqu'à donner l'impression qu'ils ne peuvent plus les contenir. Une joie sensuelle illumine leurs traits. Leurs dents brillent. Les yeux s'écarquillent. La sueur du plaisir passionné baigne leurs faces luisantes... Ce n'est 
pas une contredanse, c'est le galop de l'Opéra. Ce n'est plus un bal d'hommes mais un sabbat de possédés $»^{4}$.

De manière répétée, les auteurs condamnent « les nègres qui pervertissent notre beau quadrille français... ces «zambas » et « zambos $»^{5}$ qui déforment notre musique »... « Entre les mains de ces gens, nos mélodies sont comme saccagées », soulignent-ils. "Ne savent pas jouer »... "sauvages» sont les expressions rencontrées le plus fréquemment. La dominante populaire, populachero, arrabalero, mestizo, cholo ${ }^{6}$, est très souvent mentionnée. Les lieux mêmes sont toujours très clairement identifiés : cabarets, bouges, cafés, maisons de plaisir. La promiscuité sexuelle, le mélange de « races » sont également stigmatisés de façon péjorative. Les ports demeurent les lieux privilégiés de cette fermentation culturelle et reviennent en permanence comme lieux d'intense pratique musicale ${ }^{7}$ : La Havane, Lima, Cartagena, Rio de Janeiro, Montevideo, Buenos-Aires... A différentes périodes de l'histoire, chacune de ces villes jouera un rôle majeur dans la constitution des genres musico-chorégraphiques latino-américains.

En revanche, les mêmes voyageurs ne tarissent pas d'éloges sur les talents musicaux des Blancs, vantant le raffinement des salons de Lima et de Bogota, louant les belles interprétations des valses de Chopin qu'ils ont pu écouter et apprécier lors de la fête donnée par le Gouverneur. L'aristocratie blanche créole ${ }^{8}$ ne se reconnaît que dans la musique des salons des grandes capitales d'Europe qui représente pour elle les valeurs esthétiques universelles. Elle ignore superbement les métissages à l'œuvre dans les musiques populaires qu'elle dédaigne. Elle ne peut accepter la musique d'individus qu'elle domine socialement. Ces musiques, pourtant, ne cessent de se développer et de connaître un succès grandissant.

12 Cette différenciation sociale transite à travers les déterminants culturels, grâce à un subtil jeu social d'imitation et de distanciation des groupes entre eux. De fait, ces groupes sociaux ne vivent pas repliés sur eux-mêmes, mais en constante relation les uns avec les autres. En position dominante, le Blanc n'en reste pas moins attiré par l'univers métis, presque toujours synonyme de populaire, qui lui donne une liberté réprouvée pourtant par sa morale et son éducation. Rejet et acceptation, ce double jeu social se retrouve dans la culture issue du métissage, notamment dans la musique. 
Fig. 1 : Ensemble de Quitiplás. Curiepe, Barlovento, Venezuéla (1994).

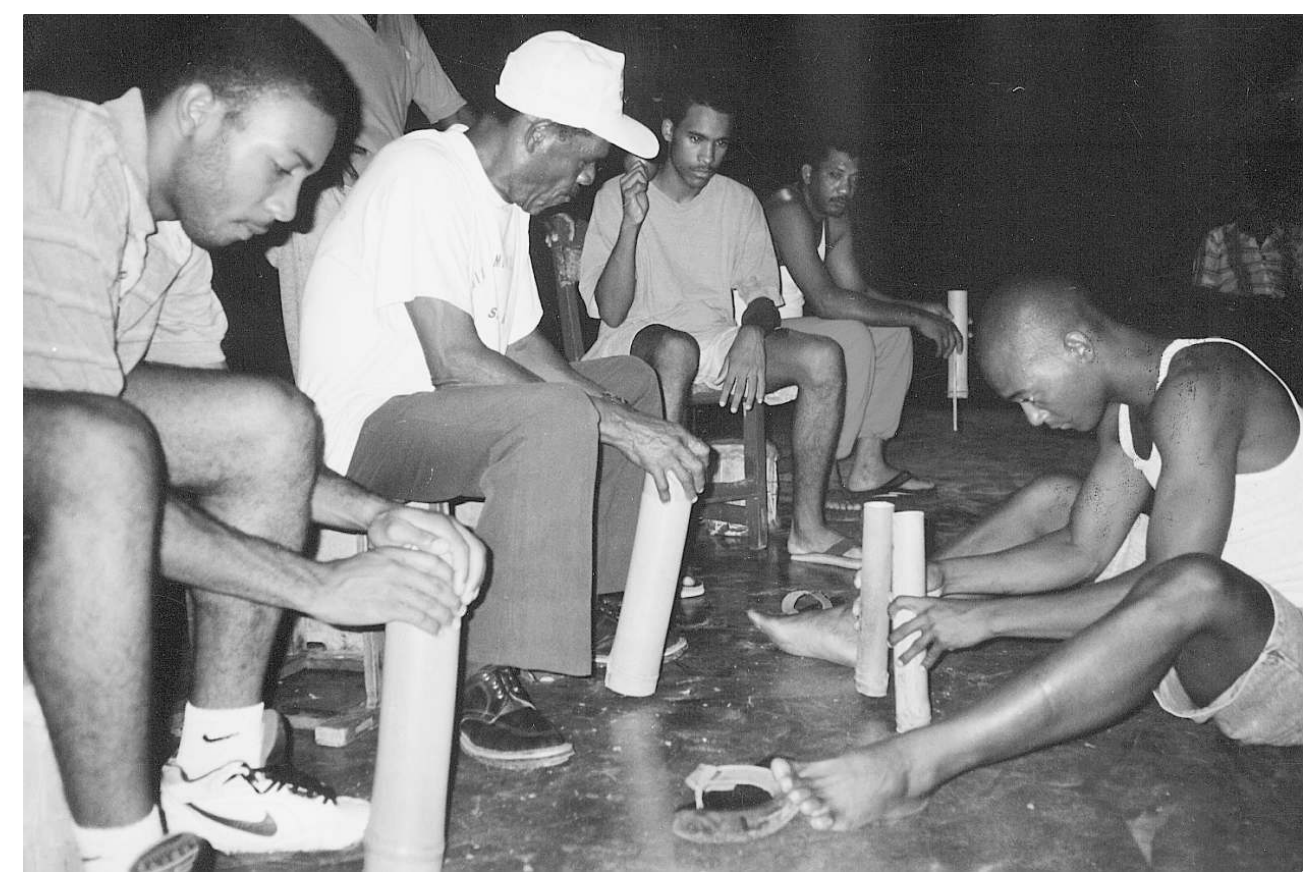

Photo : Michel Plisson barrières devant l'envahissement de son folklore et de ses fêtes. Et cela, paradoxalement, lorsque dans son désir, parfois réprimé de la Vénus noire, il accepte à son tour les danses nègres. Mais alors, il va leur faire subir des modifications pour augmenter la "distance " entre sa façon de danser à lui, et la façon dont dansent les fils de ses anciens esclaves » (1967 : 195). Horacio Salas cite Ventura Lynch ${ }^{9}$ : «la milonga en tant que danse a été inventée par les compraditos ${ }^{10}$ dans le but de se moquer des bals que donnaient les Noirs dans leurs « sociétés » (cabildos)».

Durant les carnavals du début du siècle à Buenos-Aires, les participants blancs se badigeonnent le visage de cendres (disfrazados de negro), les orchestres de rue comparsas ou murgas ${ }^{11}$, se nomment "sociedad los Negros". "Se moquer ", n'est-ce-pas précisément créer la distance sociale tout en reconnaissant l'attirance de l'autre? Ces quelques exemples montrent comment musique et danse peuvent représenter des enjeux de pouvoir dans le champ social et symbolique. De l'attirance et de la répulsion vis-à-vis des Noirs, donnée récurrente latino-américaine, naît la tension sociale nécessaire au métissage. d'autres enjeux apparaissent issus d'une recomposition sociale nouvelle. En retour, la société latino-américaine renvoie par jeux de miroirs son reflet à la métropole, comme l'observe Alejo Carpentier ${ }^{12}$.

L'histoire sociale du tango du Rio de la Plata ${ }^{13}$ (Buenos-Aires et Montevideo) et de ses mésaventures est de ce point de vue révélatrice de ce que la musique, comme production vivante, s'infléchit en fonction des enjeux symboliques qu'elle cristallise. Mentionné en Amérique latine dès le XVII ${ }^{e}$ siècle dans plusieurs pays esclavagistes le terme tango désigne les tambours et les pratiques festives des esclaves noirs (Cuba, Mexique...). Un «tango» 
voit le jour en Uruguay et en Argentine au début du XIX $x^{e}$ siècle. Cette musique devient un genre musical constitué en migrant vers les faubourgs et les prostibulos ${ }^{14}$ des zones urbaines populaires. Transplanté en Europe, le tango devient danse de salon à Paris. Rejeté par l'oligarchie, il s'acclimate dans les couches populaires urbaines d'Europe pour revenir à Buenos-Aires. Il s'empare alors des salles et des lieux fréquentés par les classes moyennes, au sein desquelles il acquiert une nouvelle esthétique, tout en continuant à exister sous une forme plus populaire. Plus tard, les masses laborieuses s'identifient à lui. On compte jusqu'à cinq cents orchestres de tango à Buenos-Aires au début des années cinquante! Il s'enfonce ensuite dans l'underground avec la chute de Perón en 1955, avant de réapparaître dans les années soixante, centre de nouveaux enjeux.

Ainsi que le remarque M. C. Lafontaine dans son étude sur la musique de Guadeloupe, une double stratégie contradictoire se met à l'œuvre entre dominants et dominés : « celle des premiers qui est d'exclure tout en instituant une norme «idéale » à laquelle répond celle des seconds qui tend à vouloir s'opposer, tout en s'intégrant. Et c'est précisément dans cette relation dialectique de la résistance et du changement entre les groupes humains que réside la formation des cultures » (1983 : 2127).

18 Les syncrétismes musicaux trouvent leur fondement dans cette double contradiction où ils dépassent leurs cultures d'origine pour s'intégrer à leur nouveau milieu et s'identifier à lui. Les genres musico-chorégraphiques latino-américains s'inscrivent dans le cadre de ce jeu social complexe. La territorialité musicale, très marquée en Amérique latine et fortement revendiquée par les musiciens eux-mêmes, procède bien souvent d'une représentation sociale sans rapport direct avec la géographie, mais qui identifie le groupe, voire la société toute entière. Certains genres musicaux acquièrent un statut quasi national comme le joropo au Venezuela, la zamba en Argentine ou le choro au Brésil, depuis longtemps déterritorialisé, mais qui conserve sa symbolique identitaire de classes moyennes urbaines. D'autres genres musicaux, en revanche, se limitent à une diffusion purement locale tout en conservant un rôle similaire de ciment social.

19 Ces genres musicaux très territorialisés sont nettement exclusifs entre eux, sauf à les mélanger par jeu musical, ce qui se produit rarement. Le groupe impose une contrainte sociale forte aux musiciens: celle de ne pratiquer que le ou les genres musicaux dans lesquels il se reconnaît.

20 Les groupes sociaux se servent souvent d'une matière musicale identique pour opérer leur différenciation. Le même genre peut adopter pour la musique et pour la chorégraphie des styles différents, l'un et l'autre se pratiquant dans des cercles sociaux distincts: l'un académique, l'autre populaire, ce dernier pouvant aussi se décliner en campagnard et urbain, ou créer un style propre à chaque village.

21 En Amérique latine, ces musiques se diffuseront par deux réseaux de sociabilité : Les fêtes publiques d'une part : processions, comparsas, carnavals...et les fêtes privées d'autre part : salons aristocratiques, bals bourgeois dans les sphères sociales élevées ou bouges urbains, maisons de plaisir, haciendas rurales et villages dans les couches populaires.

Les enjeux symboliques transcendent la musique. Ils s'exercent en profondeur sur la matière musicale elle-même en créant des tensions nouvelles sur les éléments qui la composent : champs mélodique, harmonique, rythmique, interprétatif, chorégraphique... Accentuations rythmiques moins prononcées, figures de danse moins connotées sexuellement, plus édulcorées, expressivité vocale plus retenue, phrasé moins libre, esthétique plus contenue. 

structuration mensurale de type ternaire. Précisons d'emblée que le terme de ternaire recouvre deux formules rythmiques différentes : soit une organisation ternaire avec une division binaire du temps, de type $3 / 4$, soit une organisation binaire avec une division ternaire du temps, de type 6/8. A partir de la Renaissance, les musiques exécutées pour la danse en Espagne, danses de cour ou danses villageoises, oscillent entre ces deux organisations rythmiques du temps. La danse, essentielle dans la musique de cette époque, structure formellement la plupart des musiques profanes, populaires ou savantes. Les compositeurs écrivent des "suites de danses", ensemble de pièces qui, même jouées instrumentalement, conservent leur rythmique de danse. Dans de nombreux cas, la mensuration $3 / 4$ ou $6 / 8$ n'est pas clairement définie par l'écriture musicale. Bien plus, nombre de danses intègrent des passages impliquant les deux formules. Citons-en quelques-unes, souvent populaires, acquérant parfois un statut de musique et de danse de cour : passacaille, gaillarde, chaconne, courante, sarabande, gigue. La contredanse, d'origine anglaise, fait exception, dans la mesure où elle ne définit pas un type de musique ou une structure rythmique précise, mais seulement une danse, comportant une chorégraphie au demeurant ${ }^{15}$ assez lâche.

Le $\mathrm{XVI}^{\mathrm{e}}$ siècle espagnol utilisa des musiques dotées de formules rythmiques $3 / 46 / 8$. Les œuvres des vihuelistes-compositeurs nous renseignent sur ces genres musicaux populaires, presque toujours dansés. Dans les pièces de Luyz de Narváez, Enrique de Valderrábano, Diego Pisador, Francisco Guerau, Antonio de Santa Cruz, Miguel de Fuenllana, Luis Milán, Alonso Mudarra, Santiago de Murcía, (qui vécut longtemps au 
Mexique) et dans les pièces anonymes de l'époque, est mentionné le type de danse à laquelle la musique se réfère.

Fig. 2 : Esteban García, un des maîtres de la bandola central. Caracas, Venezuéla (1996).

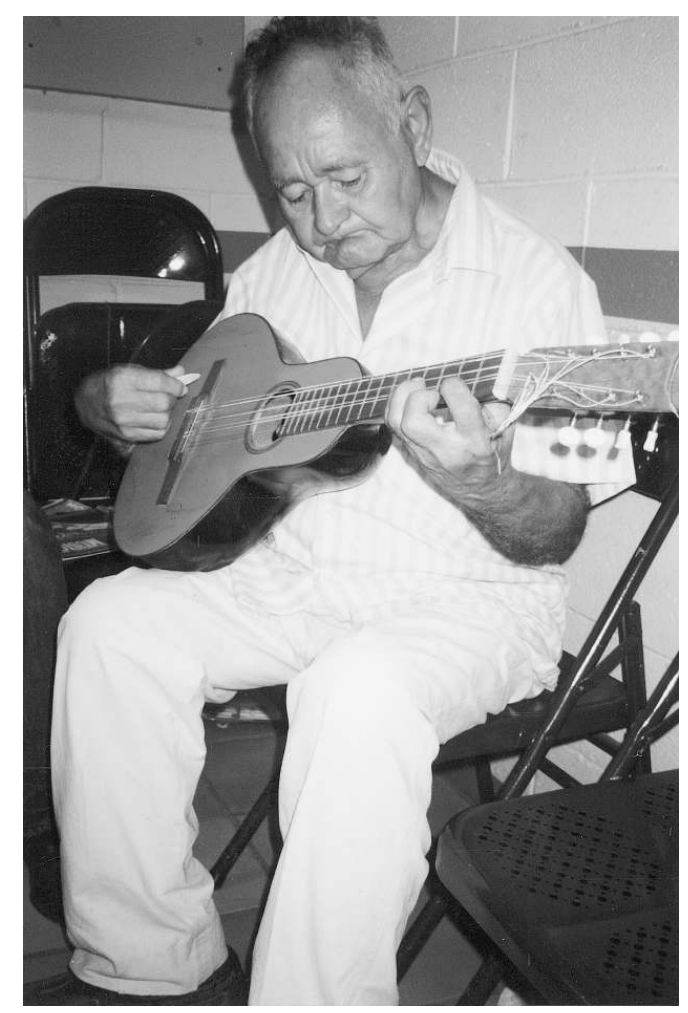

Photo : Michel Plisson.

En 1674, le célèbre vihueliste ${ }^{16}$ Gaspar Sanz publie à Salamanca son Instrvccion de mvsica sobre la gvitarra española y metodo de svs primeros rvdimentos hasta tañerla con destreza. Le sous-titre précise : Dances de Rafgueado, y Punteado, al eftilo Efpañol, Italiano, Francès, y Inglès. Aujourd'hui encore, rasgueado et punteado sont les deux techniques de jeu utilisées dans les musiques traditionnelles latino-américaines. Dans les musiques de «rasgueado et punteado ", l'interprète joue alternativement la mélodie et l'accompagnement par les doigts de la main droite qui frappent les cordes en «batterie ». Le rasgueado - sans doute italien avant d'être espagnol - (Montanaro 1983 : 15,16) marque le rythme et organise l'harmonie. Un autre musicien, ou le même, construit des lignes mélodiques en jeu de punteado. On peut décrire avec les mêmes mots un genre musical espagnol ou portugais $\mathrm{du} \mathrm{XVI}^{\mathrm{e}}$ siècle et les genres musico-chorégraphiques latino-américains traditionnels d'aujourd'hui.

Le livre de Gaspar Sanz, nous livre des pièces de forme ternaire, notées par l'auteur 3 en chiffres gras ou 6/8. Il n'y a que deux ou trois pièces à mesure binaire, allemandes ou pavanes. La référence à la danse est explicite. Elle apparaît au début de chaque pièce. On y trouve les genres suivants: jácaras, passacalle (binaire et ternaire), españoleta, paradetas, zarabanda francesa, danza de las Hachas (2/4 puis 6/8), coriente, batalla, canario... Les jacaras et canarios sont les plus nombreux. Dans le Libro segvundo figurent des gallardas, folias, matachin, chacona, maricapalos ou mariazapalos, tous genres musicaux à rythme également ternaire/binaire, de type [3/4-6/8]. 
31 Nul doute que ces genres musicaux dansés étaient accompagnés par des cordophones jouant en rasguido. De nombreuses sources le confirment. Dans un autre ouvrage ${ }^{17}$ publié en 1677, sont explicitement indiqués les rasguidos propres à chaque danse. Au-dessus des notes figure le nom de la danse se rapportant à la pièce musicale. Précédés du chiffre 3 indiquant la mesure, sont mentionnés les termes de xacaras, folias, zarabanda, mariona, matachin, rugero.

Durant la période coloniale, d'autres genres musicaux ternaires également en vigueur dans l'Espagne du $\mathrm{XVI}^{\mathrm{e}}$ au $\mathrm{XVII}^{\mathrm{e}}$ siècle s'acclimateront en Amérique tels que fandango, villancico (2/4, puis 6/8), jota... obéissant tous à la même structure rythmique fondée sur la danse.

33 A la page 11 du premier recueil de Gaspar Sanz, au-dessus d'un 6/8 très nettement indiqué, figure l'indication sesquiáltera.

\section{Les structures hémioles ou sesquialtères}

En vigueur en Europe dès l'époque médiévale, c'est au XVI ${ }^{\mathrm{e}}$ siècle en Espagne, que ces formules rythmiques trouveront leur plein développement, avant de s'acclimater en Amérique hispanophone.

Des notes sont dites sesquialtères ${ }^{18}$ ou hémioles ${ }^{19}$ lorsqu'elles sont dans le rapport de $3=2$, c'est-à-dire lorsque 3 notes sont rendues égales à 2 en durée ou l'inverse : rapport « deux contre trois ». Soit une proportion diminuante si $3=2$, soit une proportion majorante si $2=3$. Ces relations valent pour les notes comme pour les mesures. Soit deux mesures à trois temps $(223 / 4)$ combinée à trois mesures à deux temps $(3 \times 2 / 4)$ comme dans l'exemple suivant ${ }^{20}$ :

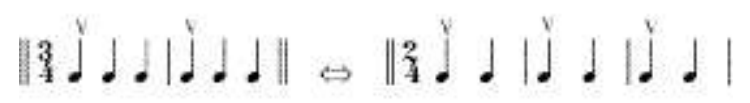

En réduisant à une mesure chacun des membres de l'expression, on a :

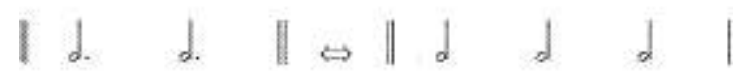

La proportion isochronique est respectée. Soit 6 noires pour un membre et 6 noires pour l'autre. Chaque mesure binaire et ternaire contient donc une durée de temps équivalente à l'autre. Ce qui change, c'est la structuration rythmique interne. Deux marquages rythmiques dans la première mesure et trois dans la seconde (si noire $=$ noire).

Or, ce rapport par lequel une mesure représente les $2 / 3$ d'une autre constitue un des principes de l'esthétique médiévale. Ces règles de proportion s'imposèrent dans la conception architecturale des monuments religieux car considérées comme parfaites, les relations $2=2$ étant jugées imparfaites ${ }^{21}$. Appliquées à la musique, ces relations hémioles se retrouvaient dans l'emploi des notes dites color, en usage dans la musique d'église dès le $\mathrm{XIV}^{\mathrm{e}}$ siècle. Colorées en rouge, certaines notes perdaient un tiers de leur valeur. En valeurs longues propres à la musique d'église, pour que les deux mesures soient équivalentes en durée, il faut donc rajouter un tiers de temps dans la mesure qui contient les notes color, soit : 


\section{$\| d . \quad d . \quad d+d$}

39 On a bien deux demies égales à trois tiers. Deux blanches pointées équivalent à trois blanches dans une proportion majorante. Soit, en valeurs brèves plus utilisées par le profane et la danse :

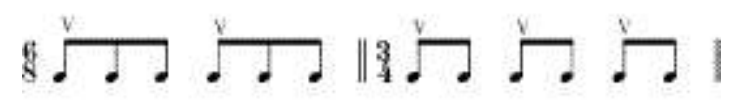

Ces successions de formules $3 / 4$ et $6 / 8$ offrent de nombreuses possibilités rythmiques. Parmi les genres populaires en usage au XVI $\mathrm{X}^{\mathrm{e}}$ siècle, jacaras et surtout canarios possédaient une structure rythmique construite sur cette alternance utilisée par de nombreux vihuélistes tels Gaspar Sanz ${ }^{22}$ ou Francisco Guerau ${ }^{23}$. La plupart des pièces publiées à cette époque contiennent ces formules, jusque dans celles trouvées en Amérique hispanique, comme le Códice Saldivar ${ }^{24}$ de León (Guanajuato/ Mexique) ou les pièces de La púrpura de la ros $a^{25}$, opéra joué à Lima en 1701 et considéré comme le premier exécuté en Amérique.

Ainsi :

41 Horizontalement :

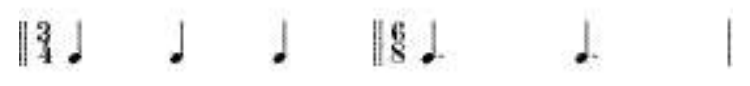

Verticalement :

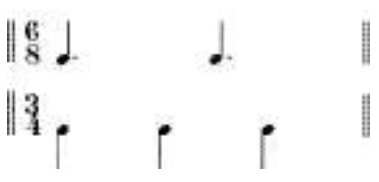

43 Le vihuéliste peut combiner seul ces formules sur son instrument, ou jouer avec d'autres musiciens. Nous obtiendrons alors des polyrythmies du type suivant :

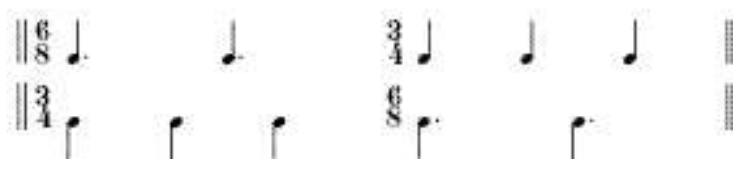

Il est maintenant impossible de déterminer lequel, du 3/4 ou du 6/8, l'emporte. En général, c'est la ligne de basse (percussions, guitare, main gauche du piano ou de la harpe) qui marque la division isochronique du temps. Cette dernière peut être binaire/temps ternaire et la ligne mélodique en 6/8. La ligne de basse peut toutefois changer brusquement et passer au-dessus. Elle peut marquer un franc $6 / 8$ durant une bonne partie de la pièce musicale, et revenir au gré du musicien au 3/4 initial, tout comme la ligne mélodique. Le jeu musical réside précisément dans l'incessante brisure rythmique. La liberté de phrasé va bien entendu de pair avec un tempo très stable, servant de contrainte à cette liberté, tous les deux nécessaires à la tension rythmique de la musique, génératrice de swing.

L'intérêt rythmique est évident : par le changement de mesure, certaines notes tombent à contre-temps. Il s'agit alors d'un nouveau système rythmique, d'une nouvelle organisation temporelle, différente de la simple addition des deux types de mesure, qui rompt avec l'organisation mensurale du temps telle que l'a pratiquée l'Europe pendant 
des siècles :

Ex :

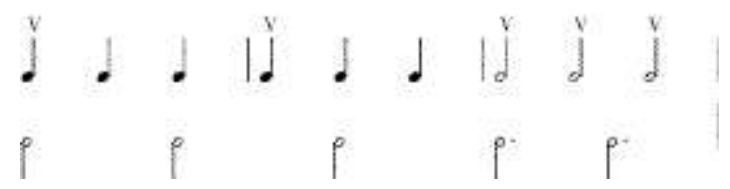

Dans ce système, quelle que soit la mesure choisie, certaines notes tomberont toujours à contre-temps. L'interprète dit: «il faut jouer binaire en pensant ternaire et jouer ternaire en pensant binaire ». De cette contradiction entre ces deux organisations temporelles irréductibles découle précisément l'extrême tension rythmique. Les appuis mélodiques, par exemple, se retrouvent systématiquement placés en dehors des temps. La raison en est le rôle particulièrement important dévolu à la musique et au rythme comme éléments constitutifs de la danse. Héritage lointain de l'Espagne baroque, elle-même peut-être tributaire de l'Afrique en ce domaine, le rythme sert d'abord à structurer la danse et ensuite seulement la mélodie. En effet, l'accentuation des notes en dehors des temps tout comme les syncopes, constituent pour le danseur de formidables stimulants, comme c'était le cas en Espagne au $\mathrm{XVI}^{\mathrm{e}}$ siècle.

Remarquons que l'utilisation de l'écriture solfégique pour noter la musique de tradition orale a suscité, en Amérique latine, notamment en Argentine, dès les premières transcriptions sur papier, de très vives polémiques dans les cercles academicos, mais aussi parmi les musiciens. Une des questions qui se posait, entre autres, était de savoir si on devait écrire les genres musicaux traditionnels en $3 / 4$ ou en $6 / 8$ ? Les connaissances acquises par les chercheurs et musicologues sur la musique ancienne et baroque espagnole depuis une vingtaine d'années ont fait perdre une grande partie de leur intérêt à ces discussions ${ }^{26}$.

Dans la tradition européenne, la mensuration de la musique marque à la fois la division isochronique du temps et l'accentuation rythmique. Ainsi, une mesure à 6/8 implique non seulement une division ternaire du temps, mais un cycle de deux temps appuyés dont le premier est fort et le second faible. La danse utilise ces deux appuis rythmiques sur le temps qui servent aussi de marquage mélodique et harmonique, comme dans la sicilienne, danse très populaire $d u$ XVIII ${ }^{e}$ siècle :

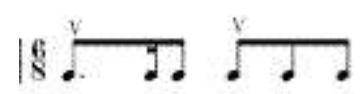

49 A l'instar du jazz, la tradition orale en Amérique latine rompt le lien entre mesure et rythme si étroit dans la musique européenne. Les temps forts ou faibles de la tradition occidentale ne deviennent pour le musicien latino-américain que des marquages parmi d'autres, servant de repère isochronique, mais sans importance majeure pour le rythme ${ }^{27}$. Beaucoup plus signifiants pour lui sont les suspensions, contre-temps et syncopes, qui identifient le genre et, symboliquement, le groupe social. Ainsi en est-il du rythme de baguala ${ }^{28} \mathrm{du}$ nord-ouest argentin frappé sur la caja :

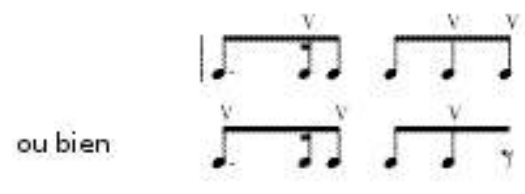



se décliner en différents styles - parfois difficiles à capter pour l'oreille non avertie sont inhérents à la structure musicale et, à ce titre, constituent un langage pour le groupe, définissant infailliblement le genre et marquant la différenciation sociale.

53 A partir de ces formules, empruntées aux vihuélistes espagnols du XVI ${ }^{e}$ et du XVII siècles dont nous ne présentons ici que le principe général, les Hispano-américains ont élaboré des musiques nouvelles, attribuant à chacune d'entre elles des marqueurs rythmiques particulièrement fins. Partant d'une structure rythmique commune, la différenciation s'est faite entre ces musiques par des signes musicaux complexes: nuances, accentuations, phrasés, tempi, retenues, glissandis, retards, rasgueos, faisant sens et langage pour les musiciens.

54 L'américanisation a résidé d'une part, dans la généralisation systématique de ces formules bi-ryhmiques d'origines européennes, et, d'autre part, sous l'influence notamment, des groupes sociaux noirs et métis, dans l'utilisation de syncopes, contretemps, accentuations, nécessaires aux danseurs, déformant les musiques d'origine et les reconstruisant pour les plier à des exigences sociales et culturelles nouvelles.

Il existe ainsi dans chaque pays, des dizaines de genres musico-chorégraphiques, possédant chacun ses caractéristiques rythmiques, sa chorégraphie, son corpus mélodique, ses pratiques instrumentales et sociales. Parmi ceux utilisant des structures rythmiques de type sesquialtère d'origine baroque européenne, on peut citer :

- Mexique : son jarocho, son huasteco o huapango, gusto, vals...

- Cuba : tonada, punto guajiro...

- Venezuela : joropo, pasaje, gaita, danza...

- Colombie : bambuco, pasillo...

- Equateur : pasillo, albazo, bomba...

- Pérou : lundú, alcatraz, marinera, vals...

- Bolivie : cueca...

- Paraguay : guarania, polka, galopa...

- Argentine : zamba, chacarera, gato, malambo, bailecito, vidala, chamamé...

- Chili : cueca, tonada...

Ainsi, en Colombie, Abadia Morales (1983: 528), ne dénombre pas moins de 61 genres musicaux/chorégraphiques traditionnels, alors même que certains genres ont été oubliés, tel le vallenato, pourtant populaire sur la côte atlantique.

Cahiers d'ethnomusicologie, 13 | 2001 
57 A la fin de l'époque coloniale, la musique de vieille tradition espagnole a presque disparu dans l'aristocratie, submergée par la musique italienne. Les émancipations nationales verront apparaître la musique de salon en provenance des capitales europénnes. Curieusement, et comme par une ironie de l'histoire, la musique des conquérants, d'abord dominante, continuera à être pratiquée dans des couches populaires socialement dominées. Cette « vieille » musique sera reléguée loin dans les campagnes et deviendra la musique "traditionnelle» d'aujourd'hui ${ }^{29}$. Avec l'émigration rurale vers les villes naissantes, elle passera directement dans les faubourgs pour s'y acclimater. Ce passage la fera changer de physionomie pour s'adapter à son nouveau milieu (contenu des textes, mélodies, phrasés rythmes...).

Dans ces brassages culturels intenses, les groupes de population noire joueront un rôle très important. La pression qu'ils exerceront sur la musique augmentera après l'abolition, lorsque les esclaves libérés commenceront à quitter les plantations pour migrer vers les villes ou lorsque les bouleversements politiques modifieront leurs conditions sociales (Haïti, Saint Domingue). Leur rôle sera essentiel dans l'américanisation des genres 3/4 6/8, mais également dans l'émergence de nouvelles cellules rythmiques à l'origine des genres musicaux afro-latino-américains.

59 L'analyse de la structure rythmique de ces musiques, jouées bien souvent de nos jours par des musiciens blancs, fait apparaître une forte parenté entre ces genres musicaux d'origine noire. Elles peuvent être regroupées dans la famille des genres 2/4 4/4.

\section{Les syncrétismes rythmiques afro-américains}

60 Dès avant la conquête, des pratiques musicales africaines sont attestées en Espagne et au Portugal où l'on dénombre déjà de nombreux esclaves. Certains avancent le chiffre de 50000 dans la péninsule, à Valencia et à Séville. D'autres sources indiquent 150000 esclaves au Portugal entre 1450 et 1500 . Les Noirs affranchis participaient aux pratiques festives de Corpus Christi et de Moros y Christianos qui mobilisaient alors un nombre important de participants. Des termes renvoyant à la danse tels que Sarambeque et zorongo existaient en Espagne au xVII ${ }^{e}$ siècle. Les Noirs avaient une influence non négligeable sur la vie musicale publique. Le terme de paracumbé, lui aussi à résonance africaine, apparaitt au XVIII ${ }^{e}$ siècle. Persistante également la rumeur historique qui voudrait que zarabanda et fandango eussent été des genres musicaux importés des Indes Occidentales (Cuba) où ils étaient pratiqués par les Noirs, et se seraient acclimatés ensuite en Espagne et au Portugal, donnant raison à A. Carpentier pour qui durant la période coloniale, en matière de musique, la péninsule ibérique reçut plus des Amériques qu'elle ne lui donna. Des métissages musicaux afro-espagnols se sont sans doute produits dans la péninsule avant la découverte des Amériques, par les Iles Canaries, passage obligé entre Séville et les Amériques, qui dès avant la conquête, connaissaient déjà un système colonial utilisant une main d'œuvre africaine servile. En Amérique, ces syncrétismes se généralisèrent dans les musiques populaires. 
Fig. 3 : Accordage du tambour cumaco et transmission familiale. Cata, Aragua, Venezuela (1999).

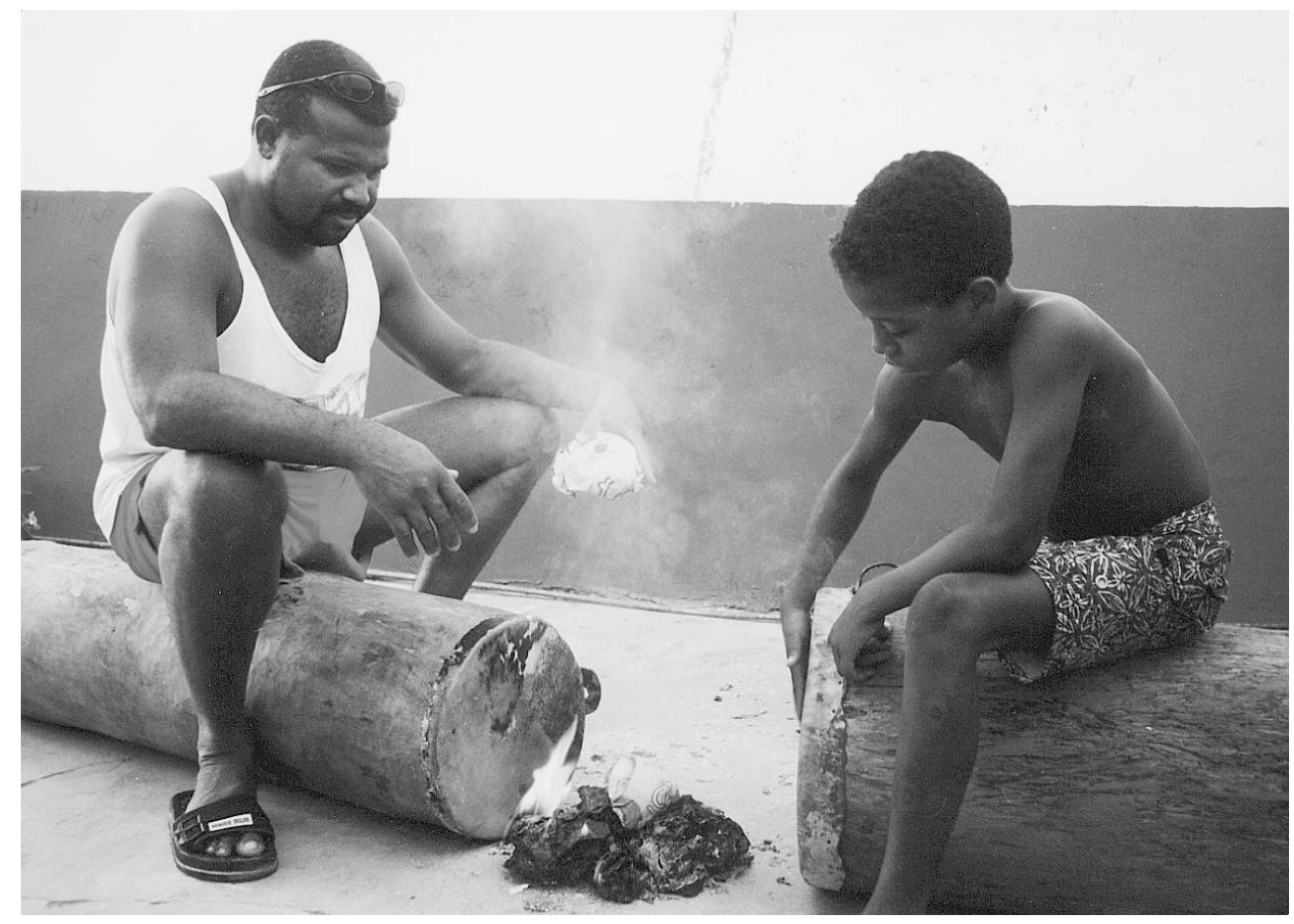

Photo : Michel Plisson

\section{Naciones, cabildos et pratique musicale}

61 Dès leur arrivée sur le sol américain, les esclaves (piezas de Indias ${ }^{30}$ ) étaient vendus et séparés de leur groupe d'origine afin de prévenir les rébellions, au demeurant, fort nombreuses ${ }^{31}$. Les Noirs bozales se regroupaient au bout de quelques mois en naciones et formaient leurs cabildos ${ }^{32}$. Les naciónes naquirent de la nécessité d'empêcher d'une part, le dépérissement des esclaves qui, isolés ethniquement, refusaient de travailler ou se suicidaient, et d'autre part, l'union de tous les esclaves contre le maître blanc. Toutefois, dans nombre de cas, les cabildos et naciones furent créés par les Noirs eux-mêmes qui y voyaient un lieu privilégié de conservation des valeurs culturelles des ancêtres et un espace de liberté ${ }^{3}$. Dans les premiers temps de la conquête, ces naciones et cofradías de tambores devinrent l'espace social où se conservèrent les rythmes anciens.

Les naciones regroupaient en général les esclaves arrivés par le même port d'embarquement, quelle que soit leur ethnie d'origine. Les esclaves Mina provenaient de l'ex-fort San Jorge da Mina (fondé par les Portugais sur l'ancienne côte des esclaves, Chano, Bénin, Nigéria), qu'ils fussent Ashanti, Ewes ou Yoruba. Les maîtres "inventèrent " les ethnies qui apparaissent sur les listas de gentilicios ${ }^{34}$ telles que bantú-congo, soudanés et guineo. Assez rapidement, les ethnies tendirent à disparaître. Les cultures « ethniques » se transformèrent en cultures "africaines » faites d'emprunts à plusieurs ethnies. Des cultures angola et congo par processus de "miscégénation ", naîtra un folklore bantú (Bastide 1967 : 16). Une culture ethnique devenait alors dominante, au sien de la nación, non sans avoir subi elle-même des influences diverses. Dans une seconde étape, on passa d'un « folklore africain à un folklore noir » (1967 : 184), telles les cultures Yoruba à Cuba et au Brésil, ou bantú au Vénézuéla et en Uruguay. Les syncrétimes religieux émergèrent 
alors, intégrant des éléments amérindiens, espagnols, français ou portugais. Ils formèrent les rituels afro-latino-américains : santeria, candomblé, vaudou, vivant de leur vie propre, détachés de l'ethnie qui les avait engendrés, assimilant des éléments exogènes à la culture d'origine, pouvant intégrer des Métis, voire des Blancs dans leurs cérémonies et leurs musiques. Selon Roger Bastide, "L'Amérique offre ainsi l'extraordinaire tableau de cultures qui se détachent de l'ethnie » (1967:15).

La composition démographique actuelle des villes d'Amérique latine ne correspond pas à ce qu'elle fut pendant des siècles. Durant toute la période coloniale jusqu'aux lois abolitionnistes $\mathrm{du} \mathrm{XIX}^{\mathrm{e}}$ siècle, les Noirs conservent des organisations communautaires puissantes dans des lieux où ils sont parfois aussi nombreux que les Blancs. Les naciones jouent un rôle important dans la vie publique des villes coloniales. Au Pérou, on dénombre à cette époque 20000 Noirs dans la seule ville de Lima, lesquels constituent $50 \%$ de la population. Dans cette même ville, on compte au xix siècle une dizaine de naciones: Angola, Caravelis, Mozambiques, Congos, Terrasnovas, Lucumies, Cambundas, mucangas ... Au Venezuela, les listas de gentilicios des archives coloniales donnent une trentaine de naciones. En Uruguay, Lauro Ayestarán (1953: 51) établit une liste des naciones qui fleurissaient à Montevidéo autour des années 1870 avec leur roi, leur reine et leur territoire urbain : Mina Nagó ( calle Joaquin Rebena ; Rey : Maria Rosso de Barboza). Congo Mina, Lubolos, Mina Magi, Mina Nucena. Les Mozambiques habitaient le barrio del cordón. Vers 1830, la nación Congo elle-même était divisée en six «provinces »: Gunga, Guanda, Angola, Mongolo, Basundi, Boma.

Lorsque les naciones étaient organisées et leurs membres nombreux, elles pouvaient négocier avec le pouvoir la pratique de leurs rites et l'usage des tambours. Toutefois, avec une constance révélatrice, on trouve dans les archives coloniales des édits émanant des autorités municipales limitant ou interdisant l'usage des tambours lors des carnavals et autres fêtes publiques. A Cuba, au Venezuela, en Colombie, au Brésil, en Argentine et au Pérou, ne sont pas rares les documents qui limitent ou interdisent la pratique musicale des Noirs dans la vie sociale. A Montevideo, Ayestarán (1953: 68) apporte quelques lumières sur ces pratiques musicales et sur l'origine du tango, tellement espagnol selon Carlos Vega. En 1807 un édit municipal est proclamé concernant les «tambos bailes de negros " par lequel ils « sont absolument interdits à l'intérieur comme à l'extérieur de la ville et on imposera à quiconque contreviendra la punition d'un mois aux œuvres publiques $»^{35}$. De nouveau en 1816, « on interdit à l'intérieur de la ville les fêtes connues sous le nom de tangos et elles sont permises seulement à l'extérieur des murs les jours de fêtes de la fin de l'après-midi jusqu'au coucher du soleil $»^{36}$. Malgré ces interdictions, les Noirs continuent leurs pratiques musicales, car la presse se plaint de «los negros con sus tangos", et les édits de la police continuent d'affirmer que dorénavant «Les fêtes dénommées candombe avec l'usage du tambour... sont interdites à l'intérieur de la ville, et seulement permises devant la mer ${ }^{37}$ (Ayestarán 1953 : 72). Plus loin, en 1888, « chaque nación avait sa parcelle de terrain pour jouer le tango, au son de la tambora, du tamboril et de la marimba $»^{38}$. Notons ici l'usage de la marimba, et d'autres idiophones disparus depuis des pratiques musicales afro-uruguayennes.

Ces Noirs constituent une menace voilée pour les autorités dominantes qui réagissent par un certain raidissement devant cette masse d'esclaves libérés depuis peu et représentant une proportion toujours croissante de la population urbaine: en Uruguay, $24 \%$ de la population en 1819 (Ayestarán (1953: 58). Cette politique est cependant doublée d'une relative tolérance devant ces manifestations qui constituent malgré tout une certaine 
mise en ordre sociale par le biais de la musique et de la danse. Quoiqu'il en soit, nous partageons l'opinion de M.C. Lafontaine (1983: 2138) selon laquelle les édits d'interdiction ne visaient pas tant les tambours que les rassemblements de Noirs qui, par leur nombre, pouvaient représenter une menace.

Dans d'autres cas, interdiction des manifestations festives et répression seront appliquées plus strictement. Elles feront disparaitre beaucoup de traits culturels africains. Ainsi, les tambours interdits par l'Église seront remplacés par le cajón dans la culture afropéruvienne. Au Venezuela, ils seront interdits dans les processions et défilés dès le XVIII siècle, mais se conserveront dans les campagnes. A Corrientes, en Argentine, les Noirs, rassemblés dans le quartier camba-cua utilisaient les tambours pour célébrer San Balthazar. De nos jours, il n'y a plus ni Noirs ni tambours à Corrientes, ni nulle part ailleurs en Argentine. Parmi les causes invoquées, qui font encore l'objet de polémiques, relevons les guerres intestines du siècle passé où les Noirs servirent en première ligne contre la promesse de leur libération, et le «blanchiment" rapide de la société, submergée par les énormes vagues d'émigrants européens. Les Noirs ont quasiment disparu en Argentine, mais ils ont laissé de profondes empreintes dans la musique et la danse : tango, milonga, rasguido doble, aujourd'hui joués et dansés par les Blancs.

L'influence des Noirs se fera sentir sur l'ensemble des musiques américaines de tradition orale ${ }^{39}$ jusqu'à la Nouvelle-Orléans ${ }^{40}$, où se pratiquait la danse congo (Carpentier 1985 : 65) avant de se répandre sur tout le continent, nord et sud, surtout du côté atlantique.

En Amérique latine, les groupes de population noire et métisse exercèrent une profonde influence sur l'ensemble des musiques de tradition orale. Après l'émancipation et l'affaiblissement du rôle social des naciones, nombre de cofradías se disloquèrent ou dépérirent. On retrouva alors les Noirs comme musiciens professionnels ou maitres de ballet dans les salons blancs.

De façon générale, l'influence noire dans les métissages musicaux latino-américains a été largement sous-évaluée, voire même occultée. Pour nombre de Latinos-américains, qu'ils soient musiciens, chercheurs ou non, l'influence noire est surtout reconnue dans les genres considérés comme «noirs " parce que pratiqués par des groupes de population noire. Il leur est souvent refusé toute influence dans les autres genres musicaux pratiqués par les Blancs ou Métisses. Pourtant, dans la période qui voit la fin de l'époque coloniale et l'abolition de l'esclavage, beaucoup de maîtres de ballet et de musiciens étaient noirs ou métis. L'étude des archives comme celle des récits des voyageurs nous apprennent que d'un bout à l'autre du continent, de Cuba au Chili comme en Argentine, les Noirs sont à la croisée des chemins musicaux. Le cas du tango, parmi des dizaines d'autres, est à cet égard édifiant. Certains auteurs comme Carlos Vega ${ }^{41}$ nièrent une quelconque origine noire au tango et lui inventèrent une naissance en Espagne. Malgré la reconnaissance affichée d'une parenté commune entre milonga, habanera et tango, joués et dansés dans une zone qui coïncidait " avec la zone la plus alimentée par les couches africaines importées », il aurait été «dangereusement glissant $»^{42}(1944: 154)$ d'y voir une influence africaine! L'origine ibérique de ces danses est réaffirmée dans son essai sur le tango ${ }^{43}$ (1936:231) et dans l'ensemble de son œuvre. La question de l'origine noire du tango fut posée à Isabel Aret $^{44}$ lors d'une interview effectuée à Caracas en 1993 ; elle entraîna de sa part une réponse péremptoirement négative, ajoutant même "es pura melodia napolitana ». Pourtant, les quatre temps du tango sont souvent accentués de la même manière que dans le ragtime et le jazz : premier temps faible/deuxième temps fort/troisième temps faible/ quatrième temps fort. On peut y voir symboliquement une inversion de l'esthétique 
blanche. On retrouve d'ailleurs cette inversion dans d'autres musiques afro d'Amérique latine. Pourtant, à Buenos-Aires, jusqu'à la fin du siècle dernier, le quartier de Monserrat était nommé el barrio del tambor à cause du «bruit » que faisaient les Noirs et leurs tambours. Carlos Vega aurait pu, dans sa jeunesse, en rencontrer dans les rues de sa ville, et en écouter dans les quartiers populaires mais il dénia aux Noirs toute influence dans l'origine du tango porteño. Le genre musico-chorégraphique malambo pratiqué dans la pampa argentine ressemble à s'y méprendre au festejo des Noirs de la côte péruvienne qui comporte aussi des mudanzas de zapateo ${ }^{45}$ sur des rythmes également sesquialtères. Sans doute, peut-on établir une relation avec le quartier malambo qui, à Lima, au XVI ${ }^{\mathrm{e}}$ siècle, était celui où habitaient les Noirs.

En fait, les Blancs empruntèrent beaucoup aux Noirs, tout en gardant une grande distance sociale, comme le montre Cuba dans sa période post-coloniale.

Fig. 4 : Tambours chimbangueles de San Benito. Bobures, Zulia, Venezuéla (1994).

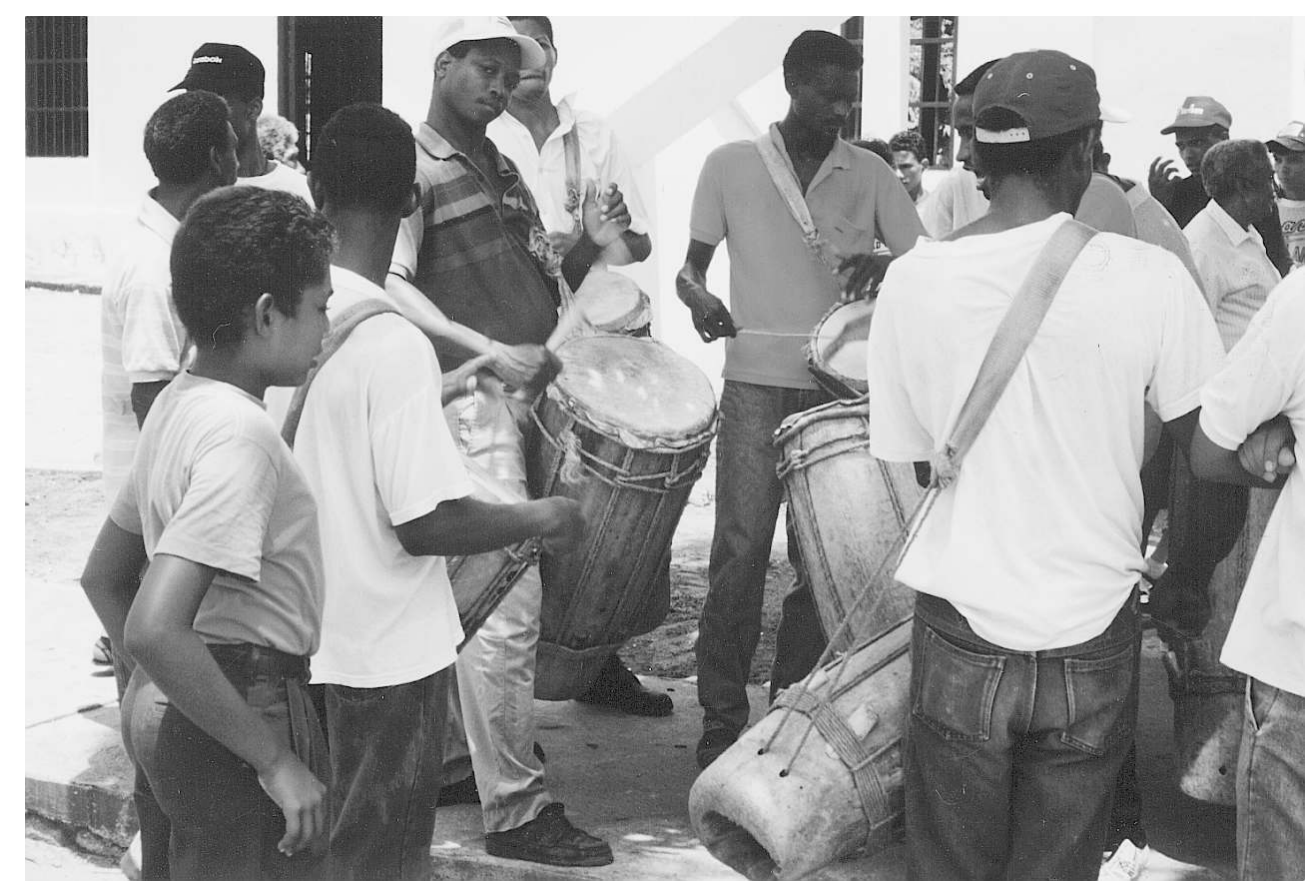

Photo : Michel Plisson

\section{Cuba : musiciens noirs et musique blanche}

71 A Cuba, Noirs et Métis occupaient un espace social particulier. De par sa situation privilégiée et son rôle central dans le commerce entre l'Espagne et les Amériques, l'île joua un rôle central dans l'élaboration de genres musicaux nouveaux de type 2/4 4/4. Cuba reçut alors toutes les influences. C'est sans doute là que les syncrétismes agirent en profondeur car les conditions y étaient très favorables de par sa position de monopole du commerce au moins jusqu'au XVII ${ }^{\mathrm{e}}$ siècle.

Pour les Grandes Antilles, Alejo Carpentier relate ce processus de jeu symbolique social à travers la musique. Après la révolte des esclaves de Saint Domingue, en 1791, les propriétaires blancs se réfugièrent à Cuba, suivis de leurs domestiques noirs. Ceux-ci, libérés de facto ou de jure, ne trouvaient à s'employer que comme domestiques ou 
musiciens. L'esclavage aboli, en effet, la société n'en restait pas moins coloniale. En haut de la hiérarchie, l'aristocratie verrouillait les emplois prestigieux et rémunérateurs. Les Noirs restaient confinés dans les emplois subalternes, gens de maison ou artisans. Le métier de musicien n'était guère mieux considéré par les Blancs. Pour le Noir, en revanche, "la musique constituait une profession très estimable, car elle se situait au sommet de ses possibilités d'ascension sociale» (Carpentier 1979/1985: 124). Le Noir choisissait la musique ou la danse, seul espace social où le Blanc ne se sentait pas en rivalité. A Cuba, dès 1827, il y a trois fois plus de musiciens noirs que de musiciens blancs, et «de 1800 à 1840, les Noirs constituèrent la grande majorité des musiciens professionnels » (Carpentier 1985 : 132). Ce processus ira s'amplifiant avec la «liberté de ventre », puis l'émancipation, qui n'est acquise à Cuba que fort tardivement, dans les années 1880 .

Ces musiciens noirs se produisaient un peu partout, dans les maisons de l'aristocratie comme dans les bals populaires où ils côtoyaient souvent les musiciens blancs. Dans les premières comme dans les seconds ils avaient accès à la musique européenne car c'est celle que les Blancs appréciaient. Les syncrétismes musicaux résulteront de cette double contradiction: les musiciens noirs exécutent la musique des Blancs qui leur permet une ascension sociale lesquels, en retour, préfèrent les Noirs pour jouer leur musique. Les Blancs, en effet, rejettent les tambours et les "tangos" de leurs anciens esclaves. L'abolition est trop récente pour que leur musique soit acceptée par le groupe social dominant. Les Noirs - et aussi les métis - s'emparent de cette musique blanche, la transforment par le phrasé, les accents, les ornements, les figures, créant des métissages qui établissent symboliquement une distance sociale entre les groupes sociaux tout en les intégrant.

Ces métissages se sont exercés notamment autour de cellules rythmiques nouvelles, produits symboliques d'enjeux entre les différents groupes sociaux.

\section{Les syncrétismes de type 2/4 4/4}

Le musicologue brésilien Luis Hector Correa de Azevedo avance l'hypothèse que la formule rythmique présente dans beaucoup de musiques afro-latino-américaines peut avoir surgi du 6/8 ternaire très fréquent dans la musique espagnole (assez rare au Portugal) lorsqu'elle se trouva accompagnée par les tambours afro-américains.

Ainsi :

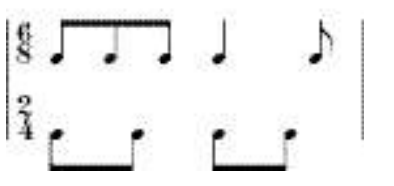

aurait donné :

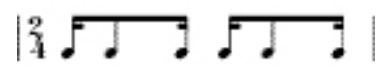

De l'irréductibilité entre ces deux systèmes rythmiques joués ensemble auraient surgi de nouvelles cellules rythmiques. De fait, les structures rythmiques ternaires étaient très présentes à Cuba dans la musique d'origine hispanique, française et aussi africaine. Fin $\mathrm{XVIII}^{\mathrm{e}}$ siècle, des bouleversements se produisent au sein de la société créole. La contredanse arrive dans les Antilles, amenée par les Français, portée par la Révolution et 
les idées nouvelles dont l'abolition de l'esclavage comme institution n'est pas la moins importante. La contredanse devient à la mode. Elle est mesurée à $2 / 4$, mais on la trouve aussi à $6 / 8^{46}$. Alejo Carpentier affirme qu'elle jouera un rôle majeur dans la formation de cellules rythmiques nouvelles. Malheureusement, son remarquable ouvrage sur la musique cubaine laisse une place réduite à la musique de tradition orale. A l'écoute de ces musiques européennes, le ternaire africain se serait binarisé selon un processus que défendent plusieurs auteurs ${ }^{47}$. En tout état de cause, une forte intensité rythmique résulte de l'assemblage de ces deux systèmes.

Cette bi-rythmie semble fonctionner comme système pour beaucoup de musique noires caraibes et non seulement à Cuba. Dans des enregistrements effectués dans la région de Barlovento, au Venezuela, zone de marronage longtemps protégée des influences extérieures par sa situation géographique et son climat inhospitalier, on entend fréquemment :

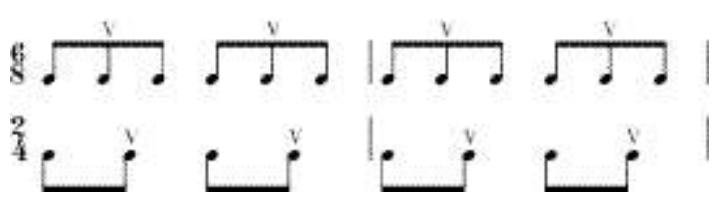

Ces structures rythmiques font pendant au système hispanisant. Alors que dans le système hispanisant, 6 croches à $6 / 8$ peuvent s'organiser verticalement sur trois noires binaires (3/4), dans le système afro-latino, 6 croches à $6 / 8$ se raccrochent à deux noires binaires ou quatre croches, c'est-à-dire à une mesure à $2 / 4$. Comme dans le système hispanisant sesquialtère, une forte tension résulte de cette bi-rythmie d'origine afro.

En effet, dans le système hispanisant, la bi-rythmie existe par l'accentuation de certaines croches par rapport à d'autres. Verticalement - qu'elles soient sur le temps ou à contretemps -, croches et noires s'exécutent conjointement à partir d'une même division isochronique du temps valable pour les parties à $3 / 4$ comme pour celles à 6/8.

81 Dans le système afroïde, si 6 croches sont divisibles par 2, elles ne le sont pas par 4, par le fait de la division binaire du temps. Autrement dit, 6 croches en haut et 4 croches en bas sont irréductibles entre elles. Pour l'oreille occidentale, la division isochronique différente du temps, entraîne une incompatibilité rythmique entre la voix haute et la voix basse. Verticalement, les croches du haut se jouent de façon disjointe par rapport à celles $\mathrm{du}$ bas. Il en résulte une tension rythmique intense, car l'espace de temps est plus serré entre les croches $d u$ haut et celles $d u$ bas. Cette bi-rythmie afro-latine contient des formules sesquialtères de 3 sur 2 , mais un sesquialtère africain et non hispanisant, puisque la base rythmique est $2 / 4$ et non $3 / 4$ ou $6 / 8$.

Cette superposition rythmique 3 sur 2, utilisée et développée avec de multiples variantes par les Noirs cubains se retrouve dans la musique africaine dans laquelle l'empilement des rythmes et l'organisation multilinéaire des voix est d'un usage fréquent. On peut émettre l'hypothèse que certains métissages ont pu intervenir par la superposition de ces deux lignes rythmiques. Dès lors, des marqueurs rythmiques peu significatifs le deviennent, du fait de la contradiction rythmique entre les deux voix, qu'ils soient situés sur le temps ou à contre-temps. Dans l'exemple du tango, dont le rythme est très apparenté à ces formules, on bascule dans une organisation rythmique inversée par rapport à la tradition occidentale. Le temps - fort ou faible - perd sa fonction de marquage rythmique et mélodique. Ici, les marquages rythmiques se placent en dehors du temps. 
Ainsi, on trouve souvent la formule rythmique suivante :

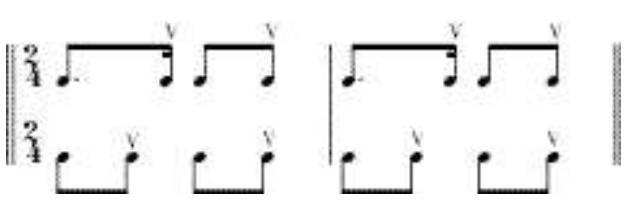

84 C'est le rythme de habanera. En haut, les deux marquages rythmiques importants sont la quatrième double croche du premier temps, et la deuxième croche du second temps, plus accentuée encore que la double croche précédente car préparant le temps suivant.

Cette formule est très proche de la formule canonique du chôro, genre instrumental né à Rio de Janeiro au début du siècle :

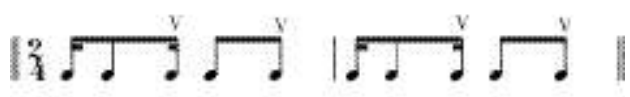

86 La répétition rapide des deux premières notes (double-croche/croche) intensifie la densité rythmique et prépare la tension sur la quatrième double-croche du premier temps. Cette dernière note joue le même rôle dans la habanera cubaine et dans le choro brésilien : elle se prolonge sur le deuxième temps, formant une syncope.

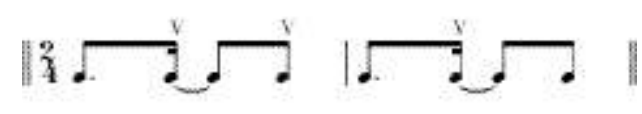

\section{Les formules afro-américaines de clave}

87 Avec cette syncope sur le deuxième temps de la mesure, nous entrons dans l'univers rythmique du tresillo cubain, bien connu des musiciens du monde entier. La musique n'est plus organisée par un temps mesuré à division binaire ou ternaire, mais par un système répartissant le marquage rythmique par groupes de deux et de trois battues sur deux mesures. Par exemple 3-3-2 (=8 pulsations isochroniques). Le marquage irrégulier du temps est au demeurant fréquent dans les musiques traditionnelles ${ }^{48}$. Ces claves rythmiques sont constitutives des musiques d'origine noire se pratiquant du côté atlantique de l'Amérique latine. Si nous considérons huit battues isochroniques, ce rythme de claves s'organise de la manière suivante :

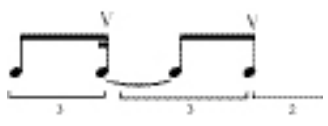

88 Nous vérifions que la formule: [croche pointée/double-croche en syncope/croche] est bien équivalente au rapport $3-3-2$ :

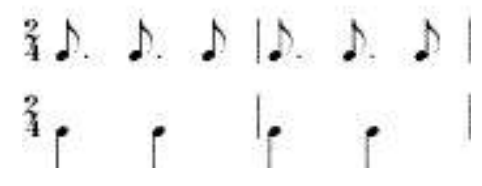

Le rapport de 3 sur 2 du tresillo cubain est encore présent. Dans la bi-rythmie latinoaméricaine d'origine afroïde, le découpage du temps est très souvent binaire à la basse. La voix mélodique peut s'articuler sur deux triolets de croches en haut, c'est-à-dire sur une division ternaire. Dans tous les cas, la basse, parfois muette et intériorisée, reste intangiblement binaire. 
90 Nous retrouvons le tresillo cubain comme cellule rythmique de base des musiques du Rio de la Plata, de la milonga et du tango. De fortes présomptions existent quant à l'origine noire de ces musiques aujourd'hui blanchies. Les milongas pampeanas des guitaristes argentins Atahualpa Yupanqui et d'Abel Fleury obéissent infailliblement à ce schéma. Les milongas urbanas des tangueros Anibal Troilo et Mariano Mores également. Les candombes des carnavals de Montevideo aussi. Plus récemment, ces formules notées : [noire pointée/ noire pointée/noire] ou 3-3-2 ont été abondamment utilisées sur quatre temps (ou deux fois deux temps) par Astor Piazzolla. Paradoxalement, ce qui pour beaucoup de musiciens tangueros des années soixante relevait d'une innovation rythmique, n'était au fond qu'un retour conscient aux sources, c'est-à-dire aux origines noires du tango.

Ainsi :

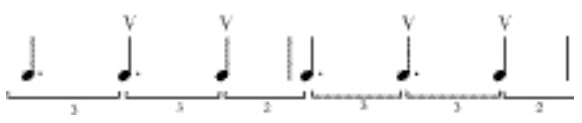

91 La quatrième double croche du premier temps de la habanera qui correspond à la deuxième noire pointée du tango piazzollien (dans un système à quatre temps) est bien la note la plus importante du système car, seule note syncopée de la mesure, c'est autour d'elle que s'organise la tension rythmique. C'est pourquoi les musiciens lui accorde une grande sollicitude. Cette attention se manifeste par une liberté de phrasé qui fait « vaciller » rythmiquement l'ensemble, rapprochant la voix mélodique d'une pulsation ternaire :

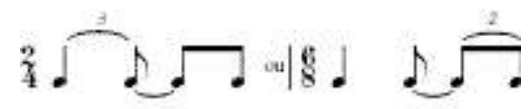

Dans cette formule, il n'existe plus la proportion 3-3-2 du tresillo cubano, puisque le premier temps est divisé par 3 et non par 4 , mais une formule approchante.

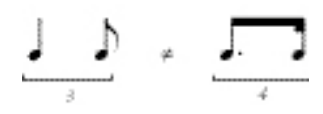

Il s'agit donc seulement d'un effet de phrasé, ce qui est confirmé par le deuxième temps qui reste toujours nettement binaire. Cette particularité rythmique réitérative des musiques d'origine noire, résulte peut-être d'une hésitation rythmique entre le ternaire européen et le binaire africain, et donc le produit d'un syncrétisme noir américain, au même titre que la blue note du Mississippi est la résultante de l'« hésitation » entre modes européens et pentatonisme africain ${ }^{49}$.

Ici, le syncrétisme n'est pas modal ni tonal, mais rythmique. Le « swing » est précisément le produit de cette « vacillation » plus ou moins grande exercée par le musicien.

A côté du tresillo cubano, on trouve très fréquemment des formules de cinquillo. Le tresillo obéit à une formule rythmique de 3 sur 2. Le cinquillo cubano à une formule de 5 sur 2. Une des formes courantes maintes fois observée et relevée entre autres par Alejo Carpentier est la suivante :

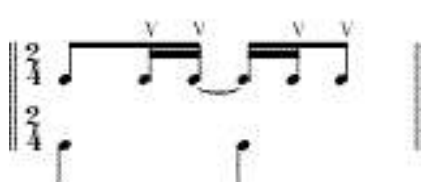



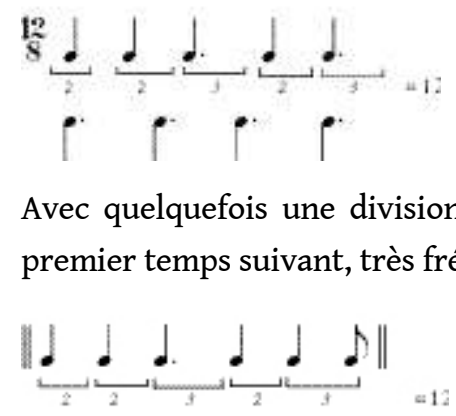
abakuás1.

Cette formule, de par la contraction de 5 notes sur 2 temps, implique nécessairement des marquages rythmiques signifiants situés en dehors du temps, facteur de tension pour les danseurs comme pour les musiciens.

Une deuxième caractéristique du cinquillo cubano est donnée par la syncope sur le deuxième temps, comme dans la habanera. Cette absence de marquage rythmique sur le second temps (dans une mesure à $2 / 4$ ), ou du troisième temps (dans une mesure à 4 temps), comme nous l'avons vu plus haut, reste une constante des musiques d'origine afro d'Amérique latine, du tango du Rio de la Plata, du choro brésilien, du candombe uruguayen, aux genres afro du Venezuela et de la musique cubaine.

Il existe bien de nombreuses possibilités d'agencer 5 pulsations sur 2 temps. La manière d'organiser ces marquages rythmiques, s'appelle la clave. Il existe deux familles de claves dans la musique caraïbe de tradition orale. La première comprend des claves à 12 pulsations isochroniques avec un comptage ternaire par temps. La deuxième famille, des claves à 16 pulsations avec une battue binaire à quatre.

Dans la musique cubaine à dominante afro, les claves à 12 temps, sous-tendues par une pulsation ternaire à la voix de basse (soit 2 mesures à $6 / 8$ par exemple), organisent le rythme selon le principe des proportions 3 et 2 , à l'instar du tresillo.

Avec quelquefois une division de la dernière unité formant comme une anacrouse du premier temps suivant, très fréquente au Venezuela.

Ces claves de 12 peuvent générer de nombreuses combinaisons rythmiques ${ }^{50}$. Ces claves ternaires sont peu utilisées dans les genres profanes, sauf dans la columbia, une des trois formes de la rumba. Ces claves ternaires à 12 , que l'on nomme parfois clave africana à Cuba, semblent être plus utilisées dans les musiques sacrées et rituelles telles que bembé, palo,

Par ailleurs, notons que ces claves autorisent une adaptation rythmique compatible avec les genres hispanophones, car décomposables en mesures binaires (de type 3/4) et/ou ternaires (de type 6/8), voire les deux à la fois.

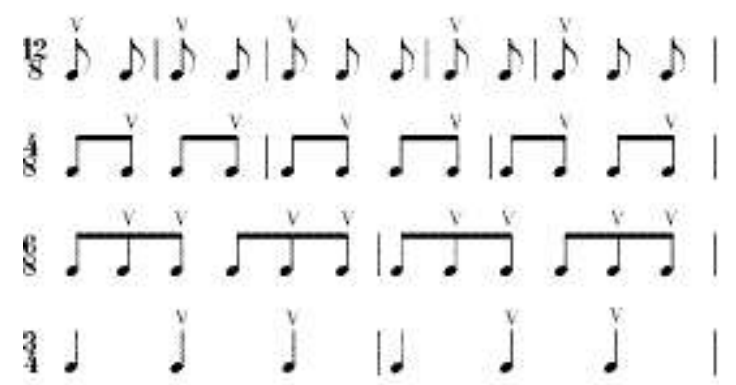

musicale superpose des rythmes hispanisants sur des rythmes afroïdes. 
105 A côté de ces claves à 12, il existe les claves à 16, dans lesquelles la pulsation marquée par la voix de basse est à 4 temps binaires (4 mesures à $4 / 4$, par exemple). Elles contiennent également de nombreuses possibilités rythmiques ${ }^{52}$. C'est sur ces claves que sont construits les genres profanes les plus connus. Parmi celles-ci, la clave de son et la clave de rumba.

106 Ainsi, la clave de rumba guaguancó ${ }^{33}$ :

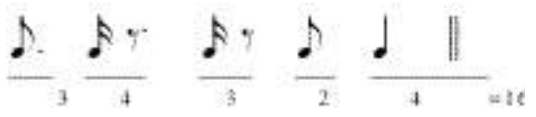

107 écrite souvent sur deux mesures :

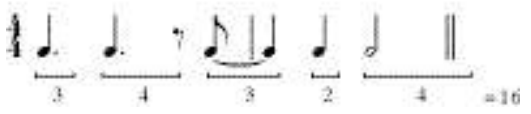

108 Les syncopes apparaissent sur le troisième temps, mais aussi sur le deuxième, comme on le voit si on écrit la même formule à $2 / 4$ :

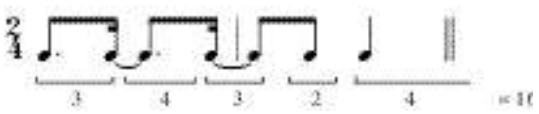

écrite parfois à $4 / 8$ :

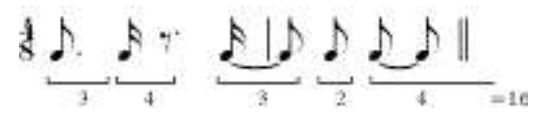

110 La clave de son, constitutive de la plupart des genres populaires cubains s'inscrit aussi dans cette logique de 16 pulsations :

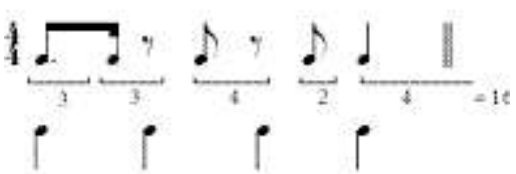

111 ou bien sur deux mesures à $4 / 8$ :

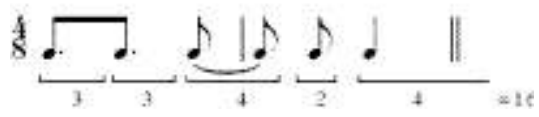

112 Ou encore à 4/4. On remarque la première mesure contient la formule canonique du tango argentin manière Piazzolla : [noire pointée/noire pointée/noire], mesure pour laquelle on trouve parfois deux noires en voix de basse. On retrouve ainsi le tresillo cubain (3 notes sur 2 dans la même durée isochronique) :

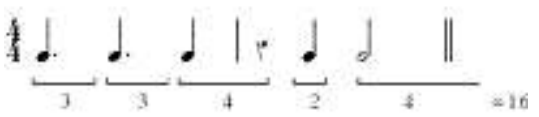

113 écrit parfois à 2/4, rendant apparentes les syncopes :

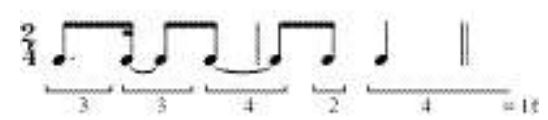

114 La première mesure correspond à la forme canonique de la habanera. Dans cette clave de son, la tension rythmique est signifiante également pour le danseur. En valeurs longues ou 
brèves, elle résulte de ces cinq notes syncopées, frappées sur une ligne de basse marquant une battue régulière de deux. Ici, la ligne de basse peut être muette, elle n'en est pas moins sous-jacente pour les musiciens.

En observant les deux portées, on s'aperçoit que la distinction entre la clave de rumba et la clave de son ne repose que sur une seule note déplacée d'une double croche vers la droite pour la rumba.

116 On perçoit ainsi l'importance du rythme dans la structuration du genre musicochorégraphique latino-américain. Une seule note déplacée et, sans aucune ambiguité pour les musiciens, le genre musical tout entier bascule.

117 Les musiques contenant ces formules rythmiques pratiquées à l'origine par les populations noires, se retrouvent un peu partout du côté atlantique et sur la côte nord du Pacifique du continent latino-américain. Ces modèles ont une parenté commune. Elaborés à partir de cellules bi-rythmiques, Cuba en fut sans doute l'épicentre avant qu'ils ne se répandent dans les zones continentales, notamment côtières. Parmi les centaines de genres existants contenant ces formules, on peut citer :

- Mexique : bamba

- Panama : cumbia, tamborito

- Cuba : guajira, danzón, són, rumba, contradanza, bolero

- Porto Rico : aguinaldo, guaracha, plena

- Saint Domingue : merengue

- Venezuela : calipso

- Colombie : currulao,cumbia,vallenato

- Brésil : choro, samba, baiaõ, frêvo, maxixe

- Pérou : festejo, son de los diablos, panalivio, danza

- Uruguay : candombe, milonga

- Argentine : rasguido doble, tango, milonga pampeana, milonga urbana...

118 Dans cette étude, nous avons essayé de montrer comment ont pu naître, en Amérique latine, certains syncrétisme rythmiques comme produits d'enjeux symboliques identitaires entre groupes sociaux. Ces tensions, cristallisées dans les métissages culturels, ont produit des patrons rythmiques nouveaux. De par leurs structures, ils ont permis une grande diversité de genres, développant sur plusieurs siècles une prolifération intense et variée dans le champ des musiques de tradition orale, chaque genre musical entretenant avec la danse une relation univoque. Ces relations rythmiques agissent au plus profond de la culture et de l'inconscient de l'individu. Elles constituent la contrainte intériorisée à partir de laquelle le musicien développe sa propre créativité, tout en gardant le lien musical profond qui l'identifie au groupe social.

BIBLIOGRAPHIE

ABADIA MORALES Guillermo, 1983, Compendio general de folklore colombiano. Bogotá : Biblioteca Banco Popular. 
ACOSTA Leonardo, 1982, Música y descolonización. La Habana : Editorial Arte y Literatura.

ANDRADE Mario de, 1989, Dicionário musical brasileiro. Rio de Janeiro : Ministério da Cultura.

AYESTARÁN Lauro, 1953, La música en el Uruguay. Montevideo : Servicio Oficial de Difusion Radio Eléctrica.

BASTIDE Roger, 1967, Les Amériques noires. Paris : Payot.

CARPENTIER Alejo, 1979, La musique à Cuba. Paris : Gallimard.

CLER Jérôme, 1994, « Pour une théorie de l'aksak ». Revue de musicologie. Paris : 181-210.

CLER Jérôme, 1998, Musique et musiciens de villages en Turquie méridionale. Thèse de doctorat de l'Université de Paris X-Nanterre. 361 p. Laboratoire d'ethnomusicologie UMR 9957.

COLLIER James Lincoln, 1981, L'aventure du jazz. 2 vol. Paris : Albin Michel.

D’HARCOURT Raoul, 1925, La musique des Incas et ses survivances. Paris : Librairie orientaliste Paul Geuthner.

DOUCET Vincent, 1989, Musiques et rites afro-américains. Paris : L'Harmattan.

FERREIRA Luis, 1997, Los tambores del candombe. Montevideo : Ed. Colihue-Sepé.

GARCÍA JIMENEZ Francisco, 1965, Así nacieron los tangos. Buenos Aires : Ed. Losada.

GASNAULT François, 1992, «Bals ou bacchanales ? Les sources de l'histoire de la danse sociale à Paris de 1830 à 1870 ». Cahiers d'ethnomusicologie régionale du Conservatoire occitan (Toulouse).

GUILCHER Jean Michel, 1969, La contredanse et les renouvellements de la danse française. Paris : Ecole Pratique des Hautes Etudes / La Haye : Mouton.

GUILCHER Jean Michel, 1984, « Le domaine du rigodon : une province originale de la danse ». In : Le monde alpin et rhodanien.

GUILCHER Jean Michel, 1995, La tradition populaire de danse en Basse-Bretagne (1963-1976).

Douarnenez : Coop Breizh/ Chasse-Marée/Armen.

HARNONCOURT Nikolaus, 1984, Le discours musical (1982). Paris : Gallimard.

LAFONTAINE Marie-Céline, 1983, Le Carnaval de l'« autre ». Les Temps Modernes (Paris) nos 441-442.

LEÓN Argeliers, 1981, Del canto y el tiempo (1974). La Habana : Editorial Pueblo y Educación.

LEYMARIE Isabelle, 1993, La salsa et le latin jazz. Paris : PUF, collection « Que-sais-je ? » nº 2742.

LEYMARIE Isabelle, 1996a, Du tango au reggae : musiques noires d'Amérique latine et des Caraïbes. Paris : Flammarion.

LEYMARIE Isabelle, 1996b, Musiques caraibes. Paris : Cité de la Musique/Arles : Actes Sud.

LOMAX Alan, 1964, Mister Jelly Roll. Paris : Flammarion.

MONTANARO Bruno, 1983, Guitares hispano-américaines. Aix-en-Provence : Edisud.

NATALE Oscar, 1984, Buenos-Aires, negros y tangos. Buenos-Aires : Editorial Peña Lillo.

ORTIZ Fernando, 1984, La clave xilofónica de la música cubana. La Habana : Editorial Letras cubanas.

ORTIZ Fernando, 1985, Los bailes y el teatro de los negros en el folklore de Cuba (1981). La Habana : Editorial Letras cubanas.

ORTIZ Fernando, 1993a, La africanía de la música folklórica de Cuba. La Habana : Editorial Letras cubanas. 
ORTIZ Fernando, 1993b, Etnia y sociedad. La Habana : Editorial de Ciencias sociales.

ORTIZ Fernando, 1993c, Travesía y trata negrera. La Habana : Publicigraf.

PACQUIER Alain, 1996, Les chemins du baroque dans le Nouveau Monde. Paris : Fayard.

PÉREZ FERNÁNDEZ Rolando Antonio, 1987, La binarización de los ritmos ternarios africanos en America Latina. La Habana : Casa de las Américas.

PLAYFORD John, 1984, The English Dancing Master, or Plaine and easie Rules for the Dancing do Country Dances, with the Tune to each Dance. London : Edit. Dance Books Ltd. (Dix-sept éditions entre 1651 et 1728).

PLISSON Michel, 1986, « Un genre musical du nord-ouest argentin : la baguala ». Journal de la Société des Américanistes LXXIII : 219-241.

PLISSON Michel, 1998, « Les genres musicaux chorégraphiques : syncrétismes originaux des musiques traditionnelles d'Amérique latine ». Musiques d'Amérique latine. Actes du colloque des 19 et 20 octobre 1996. Cordes-sur-Ciel (Tarn) : CORDAE / La Talvera : 133-153.

RAMÓN Y RIVERA Luis Felipe, 1971, La música afrovenezolana. Caracas : Ed. Universitaria.

REID ANDREWS George, 1990, Los Afroargentinos de Buenos-Aires. Buenos-Aires : Editiones de la Flor.

SALAS Horacio, 1986 Le tango. Arles : Actes Sud.

STUDER Elena F.S. de, 1984, La Trata de Negros en el Rio de la Plata durante el siglo XVIII. Buenos-Aires.

UNESCO (ouvrages collectifs)

1970, Introducción a la cultura africana en América latina. Paris : UNESCO.

1979, La traite négrière $\mathrm{du} \mathrm{XV}^{\mathrm{e}}$ au XIX $\mathrm{X}^{\mathrm{e}}$ siècle. Paris : UNESCO.

1984, L'Afrique en Amérique latine. Paris : UNESCO.

1984, «Ethnonymes et toponymes africains ». In : Histoire générale de l'Afrique. Paris : UNESCO.

VÁSQUEZ RODRÍGUEZ Rosa Elena, 1982, La práctica musical de la población negra en Perú. La Habana : Casa de las Américas.

VEGA Carlos, 1936, Danzas y canciones argentinas : teorias e investigaciones. Un ensayo sobre el tango. Buenos-Aires.

VEGA Carlos, 1944, Panorama de la música popular argentina. Buenos-Aires : Ed. Losada.

VEGA Carlos, 1956, El origen de las danzas folklóricas. 3ra edición. Buenos-Aires : Ricordi América.

\section{NOTES}

1. Une version abrégée du présent article a paru dans Musiques d'Amérique latine. Actes du colloque. Octobre 1996. C.O.R.D.A.E./La Talvera. Cordes s/Ciel (Tarn/France).

2. Vega 1956. Voir la note 29.

3. «los negros, sobre todo, desnaturalizan las danzas graciosas y apasionadas del Perú, introduciendo en ellas las posturas grotescas y los impulsos desordenados de sus bambulas africanas » (Vega 1956 : 15).

4. "Pero, la música, cada vez más viva, y sus propios movimientos, los embriagan. Sus miembros se agitan hasta dar la impressión de que ellos no pueden contenerlos. Una alegria sensual ilumina sus facciones. Sus dientes brillan; los ojos se les saltan. El sudor del placer apasionado baña sus caras relucientes... Esto no es 
una contradanza, es el galop de la Opera. Esto no es ya un baile de hombres, es un "sábado" de poseídos " (Vega $1956: 15)$.

5. «Zambas », « zambos » : Métis issus de l'union de Noirs et d'Indiens.

6. Populachero : populacier. Arrabalero : issu des faubourgs. Mestizo : métis issu de Blanc et de Noir. Cholo : Au Pérou, métis issu de Blanc et d'Indien.

7. La Nouvelle-Orleans jouera un rôle identique pour les musiques noires d'Amérique du Nord.

8. Dans nombre de pays latino-américains, le Criollo (créole) est le Blanc né en Amérique. En Argentine, l'adjectif criollo signifie vernaculaire, typique de la zone, que l'individu soit blanc ou métisse. Dans les deux cas, le sens est différent du terme créole utilisé à la Nouvelle-Orléans et dans les Antilles et qui se réfère au métissage.

9. Ventura Linch : La provincia de Buenos-Aires hasta la definición de la cuestión capital de la República (Buenos Aires, 1883).

10. Compadrito : mauvais garçon en argot de Buenos Aires. Personnage qui est mis en scène dans l'univers social du tango. Les compadritos étaient des Blancs d'origine européenne.

11. Comparsas et murgas étaient des ensembles festifs (orchestres de rue, groupes de danseurs de carnaval).

12. «Il est un fait certain: les danses primitives apportées de la Péninsule acquéraient une nouvelle «physionomie en Amérique, quand elles entraient en contact avec le Noir et le Métis. Modifiées dans le tempo, dans les mouvements, enrichies de gestes et de figures d'origine africaine, elles faisaient habituellement le voyage inverse, et revenaient au point de départ avec des caractères de nouveauté. Dans la chaleur des ports naissaient aussi des danses qui n'étaient que des réminiscences de danses «africaines dépouillées de leur poids rituel. Mais l'Amérique, pendant la période de formation de ses sociétés, donna beaucoup plus qu'elle ne reçut» (Carpentier $1979: 60)$.

13. Avant la fin du XIX ${ }^{\mathrm{e}}$ siècle le terme tango ne se réfère à aucune forme musicale, rythmique ni chorégraphique définie, seulement aux lieux où vivent les esclaves noirs, aux pratiques festives de ces derniers et au bruit que font leurs tambours.

14. prostíbulos : maisons closes.

15. Selon divers auteurs, la contredanse française, danse des classes roturières, serait à l'origine de la contradanza à Cuba. Les mêmes remarques peuvent s'appliquer pour cette dernière. La musique et la danse de la contradanza ne sont guère définis. Il existe des partitions à $2 / 4$, d'autres à 6/8. Si la contredanse française a apporté quelque chose à la musique cubaine, c'est peut-être par ses mélodies, sans doute par ses pratiques sociales de danse, certainement pas par sa structure rythmique (cf. Carpentier 1979 : 61). Les mélodies, quant à elles, sont accompagnées à la main gauche du piano par un rythme de croche pointée/double croche/deux croches. Soit le rythme de habanera (appelé alors à Cuba rythme de tango). C.f. voir également note $\mathrm{n}^{\circ} 42 \mathrm{~J} . \mathrm{M}$. Guilcher (1969).

16. vihuela et guitarra sont alors assez proches l'une de l'autre. Seul le nombre de chœurs et les pratiques sociales les différencient.

17. Luz y norte musical para caminar por las cifras de la guitarra. Compuesto por Don Lucas Ruiz de Ribayaz y Foncea ». Madrid. Melchior Alvarez año 1677. Voir également «Compendio numeroso » du harpiste Diego Fernández de Huete (1702).

18. Sesquialtère : du latin sesqui : une fois et demi et alter l'autre. Une fois et demi l'autre.

19. Hémiole : du grec hémiolios : un et demi. Les rapports $3 / 2$ s'appliquent ici aux rythmes. Dans les calculs d'intervalle, le rapport hémiole $3 / 2$ est aussi celui de la quinte pythagoricienne.

20. Le $\mathbf{v}$ au-dessus de la note signifie que la note est accentuée. $\mathbf{V}$ si elle est très accentuée.

21. La mystique médiévale a largement puisé dans les théories de Boèce (480-524) qui développe dans son œuvre De Institutione Musica l'idée selon laquelle « la musique n'est pas autre chose que la science des nombres qui régissent l'harmonie du monde ».

22. Cf. CD Folias et canarios, par Hesperion XX \& Jordi Savall. Astrée E 8516, 1994. 
23. Cf. CD Francisco Guerau, Poema harmonico par Hopkinson Smith, guitare baroque à cinq chœurs. Astrée E 8722, 1990.

24. Transcription J.Hinojosa/J. Cardoso. Manuscrit non édité. Cf. CD Santiago de Murcia/codex $n^{\circ} 4$, Mexico c. 1730. Ensemble Kapsberger. Dir. Rolf Lislevand. Ed. Astrée/Naïve, 2000.

25. La púrpura de la rosa du compositeur espagnol Torrejón y Velasco (1644-1728). Premier opéra composé et éxécuté en Amérique. DHM/BMBG (1999). Le baylete du harpiste Luiz de Ribayaz, qui est intégré à l'œuvre, contient la même structure harmonique que le malambo, genre musicochorégraphique de la pampa argentine : accords du I ${ }^{\mathrm{er}}$, IV ${ }^{\mathrm{e}}$ et $\mathrm{V}^{\mathrm{e}}$ degré sur deux mesures.

26. Cf. référence Nikolaus Harnoncourt. Voir notamment les passages concernant le rôle de la Révolution Française dans la création des Conservatoires de musique et de danse et la diffusion d'une musique considérée comme universelle.

27. Dans de nombreux cas, les temps forts ou faibles de la tradition européenne seront seulement déplacés. Ainsi, le tambour surdo du samba brésilien marque le second temps du 2/4 plus fort que le premier. Les temps peuvent être marqués en silence, comme dans le cas de la chacarera de la province de Santiago del Estero, en Argentine, structurée sur un cycle de trois marquages rythmiques. Le premier temps est alors presque muet ("très fort à l'intérieur », dit le musicien), le second assez fort, le troisième, très accentué. En somme, une inversion presque parfaite de la rythmique occidentale.

28. Baguala: chant des montagnes du nord-ouest de l'Argentine, utilisant un jeu d'harmoniques en voix de tête (kenko), accompagné d'un membraphone à main, la caja. Voir Plisson 1986.

29. Certains genres musicaux connaissent une structure très proche de la musique jouée en Europe à l'époque baroque. Cf. livret CD Venezuela: Musique de l'Orénoque: Cheo Hurtado. Ocora/ Radio France, 1997. Enregistrements et texte M. Plisson.

30. Pieza de Indias: terme utilisé dans certains documents écrits de l'époque coloniale pour désigner les esclaves.

31. Les esclaves qui parvenaient à s'échapper se réfugiaient loin à l'intérieur des terres, formant des kilombos, ou palenques ou cumbe de cimarrones. Protégées en partie des métissages, ces microsociétés conservèrent longtemps les traditions africaines musicales ritualisées.

32. cabildo: Littéralement: conseil municipal. Les cabildos existaient déjà à Séville avant la conquête. Ils se généralisèrent dans les Indes Occidentales sous la pression du nombre.

33. Cf. : Livret CD «Venezuela : chants et tambours des confréries noires " Ocora/radio France 1995. Enregistrements et texte M. Plisson.

34. Lista de gentilicio : littéralement groupe de parenté ou de lignée, établie lors de l'arrivée des esclaves.

35. "Se prohiban absolutamente dentro y fuera de la ciudad y se impongan a quien contrabenga el castigo de un mes a las obras públicas»

36. "Se prohiban dentro de la ciudad los bayles conocidos por el nombre de tangos y solo se permiten a extramuros en las tardes de los dias de fiesta hasta puesta del sol »

37. « Los bailes denominados candombe con el uso del tambor...estan prohibidos en el interior de la ciudad, y s-los permitidos frente al mar... ».

38. « Cada nación tenia su canchita para darle al tango... al son de la tambora, del tamboril, de la marimba... ».

39. On peut fréquemment entendre dans les orchestres de jazz de cette époque, des rythmes de clave de type 3-3-2. Cf. la célèbre formation des « Hot Peppers » que dirigeait J. R. Morton.

40. C.f. J. L. Collier L'aventure du jazz. Le grand pianiste de jazz «créole de couleur » Jelly Roll Morton confiait à Alan Lomax en 1939 que toute bonne musique de jazz doit contenir une " couleur espagnole ", en fait un rythme de habanera. Collier ajoute à la page 117 : « Le rythme de habanera est très proche du rythme habituel sur lequel la majeure partie du jazz a été improvisée ou composée ». Un parallèle peut s'établir entre les conditions sociales qui présidèrent à la naissance du jazz à la Nouvelle Orléans et celles qui virent naître le tango du Rio de la Plata. J. R. 
Morton était pianiste de bordel et métis, tout comme Rosendo Mendizábal, pianiste de couleur qui jouait dans les prostíbulos à Buenos-Aires, et qui, au début du siècle, fut le compositeur d'un des premiers titres enregistrés comme tango.

41. Carlos Vega (1898-1966), ethnomusicologue argentin, commença à étudier dès avant la deuxième guerre mondiale la musique traditionnelle de son pays, notamment dans la région du nord-ouest et plus tard au Pérou. Il laisse une œuvre considérable, quoique en partie discutée aujourd'hui. Bibliographie complète parue dans Cuardernos del Instituto de Antropología $n^{\circ} 6$. Buenos-Aires 1966-1967.

42. «Con la más nutrida zona de las capas africanas importada » [...] « peligrosamente resbalizada ».

43. Pour la réfutation du tango andaluz comme origine ibérique du tango porteño, voir la brochure qui accompagne le coffret édité par l'Instituto nacional de musicología : Antología del tango rioplatense Vol I. Buenos-Aires 2 LP, 1980.

44. Isabel Aretz. Ethnomusicologue argentine née en 1913 à Buenos-Aires. Elève de Carlos Vega, comme son futur époux, l'ethnomusicologue vénézuélien Luis Felipe Ramón y Rivera. Directrice à Caracas du FUNDEF jusqu'en décembre 1994. Interview publié dans Cahiers de musiques traditionnelles 7, 1994. Le passage en question n'a pas été publié dans les Cahiers, mais figure dans l'enregistrement de l'interview.

45. «Variations de figures de danse » de malambo.

46. La contredanse est la déformation française de la country dance anglaise, introduite à la Cour de Versailles dans les dernières années du règne de Louis XIV par le maître de ballet André Lorin. La country dance n'impose aucun pas. C'est Lorin qui les formalisera pour la Cour. Malgré cela, celle-ci la dédaigne quelque peu. Ce n'est qu'avec l'ouverture de bals publics à entrée payante, en 1715, sous la Régence, que la contredanse s'imposera. Les « belles danses à deux » (c'est-à-dire les danses aristocratiques) tombent alors en désuétude, au profit de la contredanse qui permet de faire danser plusieurs couples à la fois avec une liberté plus grande dans les pas. (Guilcher 1969).

47. Pérez Fernández 1987 et Ramón y Rivera 1971.

48. Ainsi dans la musique flamenca (12 temps) et les musique d'Europe centrale et de Turquie (rythmes aksak à 7 ou 9 temps) (Cler 1994 et 1998).

49. Cf. Collier $1981: 29$ et suiv.

50. Le nombre total de possibilités rythmiques contenues dans ces formules à 12 avec des proportions de 3 et de 2 pulsations renvoie à un problème de dénombrement résolu par l'analyse combinatoire. Classer deux objets parmi cinq sachant que l'ordre des deux objets n'importe pas. Le nombre de combinaisons possible est donné par la formule

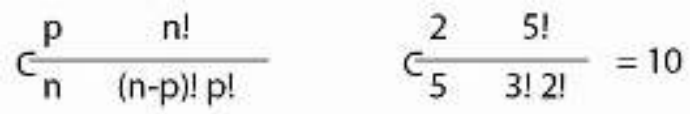

Voici ces 10 possibilités :

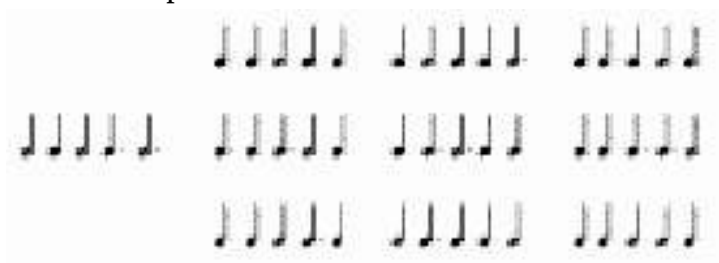

Certaines constituent des patrons rythmiques de genres musicaux, d'autres sont simplement utilisées comme variations rythmiques par les musiciens. Signalons les cas particuliers de l'aguinaldo et du merengue vénézuélien à $5 / 8$ dans lequel la répartition entre croches est isochronique mais avec une accentuation sur la $5^{\mathrm{e}}$ et la $1^{\text {re }}$ croches, accompagné bien souvent par une basse à $3 / 4$.

51. Cf. le CD Cuba: danse des dieux, Ocora HM 83, 1988. 
52. On peut dénombrer l'ensemble des combinaisons de trois objets parmi cinq. Soit :

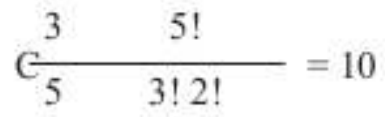

53. Cf.le CD Cuba:Celeste Mendoza, Aspic X 55516, 1993 (archives EGREM). Notamment les plages 2, $4,8,11,12,16$.

\section{RÉSUMÉS}

En Amérique latine, les métissages musicaux ne furent pas l'œuvre du corps social dans son ensemble. Les groupes sociaux utilisèrent la musique comme d'autres déterminants culturels pour s'identifier en opérant une distanciation. Cette différentiation sociale appartient à l'ordre du spectaculaire pour ce qui est de la musique et de la danse. Chaque groupe social se reconnaît dans un univers musical particulier. Or, l'unité entre danse et musique s'établit par le rythme, lien essentiel, définitoire du genre musico-chorégraphique en Amérique latine. Dans cette aire culturelle, les syncrétismes comme vecteurs d'enjeux sociaux s'expriment particulièrement bien à travers l'organisation rythmique de la musique. Produits des trois ensembles culturels amérindien, européen et africain, les métissages musicaux latino-américains se sont cristallisés essentiellement autour de trois patrons rythmiques. Dans cette étude, sont abordés ceux d'origine espagnole, et ceux d'origine afro-américaine. Résultante d'enjeux symboliques entre groupes sociaux, ces patrons rythmiques ont pu générer, grâce à leur structuration interne, des centaines de genres musicaux.

\section{AUTEUR}

\section{MICHEL PLISSON}

Michel Plisson est professeur de sciences sociales et chargé de cours en sciences économiques à l'Université Paris III/Sorbonne Nouvelle. Après l'obtention d'un DEA en espagnol à L'Université de Paris X/Nanterre, il a obtenu dans cette même Université une maîtrise d'ethnomusicologie. Il a une formation de conservatoire en guitare classique et flûte traversière, a étudié avec plusieurs guitaristes en Amérique latine (Brésil, Pérou, Argentine) et procédé à de nombreuses transcriptions de musiques traditionnelles. Il a réalisé une quinzaine de voyages d'études sur ce continent et a publié des études et articles sur les musiques traditionnelles d'Amérique latine, s'intéressant surtout à la question des origines baroques, des métissages musicaux et des enjeux symboliques auxquels ils renvoient. Collabore régulièrement à plusieurs revues musicales depuis plusieurs années. A effectué depuis trente ans de multiples enregistrements dans cette partie du monde, et publié une dizaine de CD, notamment dans la collection Ocora/Radio France (Venezuela, Argentine, Bolivie). Il prépare actuellement une thèse d'ethnomusicologie sur "Musique et société dans le nord-ouest argentin" pour Université Paris IV/Sorbonne. 